\title{
SELF-SIMILAR EVOLUTION OF COSMIC-RAY MODIFIED SHOCKS: THE COSMIC-RAY SPECTRUM
}

\author{
Hyesung KAnG ${ }^{1}$, Dongsu Ryu ${ }^{2}$, AND T. W. Jones ${ }^{3}$ \\ ${ }^{1}$ Department of Earth Sciences, Pusan National University, Pusan 609-735, Korea; kang@uju.es.pusan.ac.kr \\ ${ }^{2}$ Department of Astronomy and Space Science, Chungnam National University, Daejeon 305-764, Korea; ryu@ @anopus.cnu.ac.kr \\ ${ }^{3}$ Department of Astronomy, University of Minnesota, Minneapolis, MN 55455, USA; twj@msi.umn.edu \\ Received 2008 October 4; accepted 2009 January 20; published 2009 April 7
}

\begin{abstract}
We use kinetic simulations of diffusive shock acceleration (DSA) to study the time-dependent evolution of plane, quasi-parallel, cosmic-ray (CR) modified shocks. Thermal leakage injection of low-energy CRs and finite Alfvén wave propagation and dissipation are included. Bohm diffusion as well as the diffusion with the power-law momentum dependence are modeled. As long as the acceleration timescale to relativistic energies is much shorter than the dynamical evolution timescale of the shocks, the precursor and subshock transition approach the time-asymptotic state, which depends on the shock sonic and Alfvénic Mach numbers and the CR injection efficiency. For the diffusion models we employ, the shock precursor structure evolves in an approximately self-similar fashion, depending only on the similarity variable, $x /\left(u_{s} t\right)$. During this self-similar stage, the CR distribution at the subshock maintains a characteristic form as it evolves: the sum of two power laws with the slopes determined by the subshock and total compression ratios with an exponential cutoff at the highest accelerated momentum, $p_{\max }(t)$. Based on the results of the DSA simulations spanning a range of Mach numbers, we suggest functional forms for the shock structure parameters, from which the aforementioned form of CR spectrum can be constructed. These analytic forms may represent approximate solutions to the DSA problem for astrophysical shocks during the self-similar evolutionary stage as well as during the steady state stage if $p_{\text {max }}$ is fixed.
\end{abstract}

Key words: acceleration of particles - cosmic rays - shock waves

Online-only material: color figures

\section{INTRODUCTION}

Diffusive shock acceleration (DSA) is widely accepted as the primary mechanism through which cosmic rays (CRs) are produced in a variety of astrophysical environments (Bell 1978; Drury 1983; Blandford \& Eichler 1987). The most attractive feature of the DSA theory is the simple prediction of the powerlaw momentum distribution of CRs, $f(p) \propto p^{-3 \sigma /(\sigma-1)}$ (where $\sigma$ is the shock compression ratio) in the test-particle limit. For strong, adiabatic gas shocks, this gives a power-law index of 4, which is reasonably close to the observed, "universal" index of the CR spectra in many environments.

However, it was recognized early on, through both analytical and numerical calculations, that the DSA can be very efficient and that there are highly nonlinear back-reactions from CRs to the underlying flows that modify the spectral form as well (e.g., Malkov \& Drury 2001, for a review). In such CRmodified shocks, the pressure from CRs diffusing upstream compresses and decelerates the gas smoothly before it enters the dissipative subshock, creating a shock precursor and governing the evolution of the flow velocity in the precursor. On the other hand, it is primarily the flow velocity through the precursor and the subshock that controls the thermal leakage injection and the DSA of CRs. Hence the dynamical structure of the flow and the energy spectrum of CRs must evolve together, influencing each other in a self-consistent way.

It is formation of the precursor that causes the momentum distribution of CRs to deviate from the simple test-particle powerlaw distribution. With a realistic momentum-dependent diffusion, $\kappa(p)$, the particles of different momenta, $p$, experience different compressions, depending on their diffusion length, $l_{d}(p)=\kappa(p) / u_{s}$ (where $u_{s}$ is the shock speed). The particles just above the injection momentum $\left(p_{\text {inj }}\right)$ sample mostly the compression across the subshock $\left(\sigma_{s}\right)$, while those near the highest momentum $\left(p_{\max }\right)$ experience the greater, total compression across the entire shock structure $\left(\sigma_{t}\right)$. This leads to the particle distribution function that behaves as $f(p) \propto p^{-3 \sigma_{s} /\left(\sigma_{s}-1\right)}$ for $p \sim p_{\text {inj }}$, but flattens gradually to $f(p) \propto p^{-3 \sigma_{t} /\left(\sigma_{t}-1\right)}$ toward $p \sim p_{\max }$ (Duffy et al. 1994).

Analytic solutions for $f(p)$ at the shock have been found in steady state limits under special conditions; for example, the case of a constant diffusion coefficient (Drury et al. 1982) and the case of steady state shocks with a fixed $p_{\max }$ above which particles escape from the system (Malkov 1997, 1999; Amato \& Blasi 2005, 2006). In these treatments, the selfconsistent solutions involve rather complicated transformations and integral equations, so are difficult to use in general, although they do provide important insights. In particular, Malkov (1999) showed that in highly modified, strong, steady shocks $\left(\sigma_{t} \gg 1\right)$ with a fixed $p_{\max }$, the spectrum of CRs flattens to $f(p) \propto p^{-3.5}$ for $\kappa(p) \propto p^{\alpha}$ with $\alpha>1 / 2$. He also argued that the form of the CR spectrum is universal under these conditions, independent of $\kappa(p)$ and $\sigma_{t}$. In an effort to provide more practical description Berezhko \& Ellison (1999) presented a simple approximate model of the CR spectrum at strong, steady shocks in plane-parallel geometry. They adopted a threeelement, piecewise power-law form to represent the spectrum at nonrelativistic, intermediate, and highly relativistic energies. And they demonstrated that this model approximately represents the results of their Monte Carlo simulations.

In Kang \& Jones (2007; Paper I), from kinetic equation simulations of DSA in plane-parallel shocks with the Bohmlike diffusion $(\kappa \propto p)$, we showed that the $\mathrm{CR}$ injection rate and the postshock states approach time-asymptotic values, even as the highest momentum $p_{\max }(t)$ continues to increase with time, and that such shocks then evolve in a "self-similar" fashion. We 
then argued that the nonlinear evolution of the shock structure and the CR distribution function in this stage may be described approximately in terms of the similarity variables, $\xi=x /\left(u_{s} t\right)$ and $Z \equiv \ln \left(p / p_{\text {inj }}\right) / \ln \left[p_{\max }(t) / p_{\text {inj }}\right]$. Based on the self-similar evolution, we were able to predict the time-asymptotic value of the CR acceleration efficiency as a function of shock Mach number for the assumed models of the thermal leakage injection and the wave transportation. In those simulations we assumed that the self-generated waves provide scatterings sufficient enough to guarantee the Bohm-like diffusion, and that the particles do not escape through either an upper momentum boundary or a free-escape spatial boundary. So the CR spectrum extended to ever higher momenta, but at the same time the particles with the highest momentum spread over the increasing diffusion length scale as $l_{\max } \propto \kappa\left(p_{\max }\right) / u_{s} \propto p_{\max } \propto t$. We note that in Paper I we considered plane-parallel shocks with shock Mach number, $2 \leqslant M_{0} \leqslant 80$, propagating into the upstream gas with either $T_{0}=10^{4}$ or $10^{6} \mathrm{~K}$, since we were interested mainly in cosmic structure formation shocks.

The simplicity of the results in Paper I suggested that it might be possible to obtain an approximate analytic expression for the CR spectrum in such shocks, but the simulations presented in that paper were not sufficient to address that question. Thus, we further carried out an extensive set of simulations to fully explore the time-dependent behavior of the CR distribution in CR-modified shocks with shock Mach numbers $M_{0} \geqslant 10$. In this paper, from the results of these simulations, we suggest practical analytic expressions that can describe the shock structure and the energy spectrum of accelerated particles at evolving CRmodified shocks in plane-parallel geometry, in which the Bohmlike diffusion is valid.

In realistic shocks, however, once the diffusion length $l_{\max }$ becomes comparable to the curvature of shocks, or when the growth of waves generated by the CR streaming instability is inefficient, the highest energy particles start to escape from the system before they are scattered and advected back through the subshock. In such cases, $p_{\max }$ is fixed, and the CR spectrum and the shock structure evolve into steady states. So, for comparison, we carried out additional simulations for analogous shocks in which the particles are allowed to escape from the system once they are accelerated above an upper momentum boundary, $p_{\text {ub }}$. Those shocks achieve true steady states and the shock structure and the CR distribution become stationary with forms similar to those maintained during the self-similar stage of shock evolution. In this sense, our solution is consistent with the analytic solutions for steady state shocks obtained in the previous papers mentioned above.

In the next section, we describe the numerical simulations and results. The approximate formula for the CR spectrum will be presented and discussed in Section 3, followed by a summary in Section 4. We also include an Appendix that presents simple analytic and empirical expressions that can be used to characterize the dynamical properties of CR-modified shocks.

\section{NUMERICAL CALCULATIONS}

\subsection{Basic Equations}

In our kinetic simulations of DSA, we solve the standard gasdynamic equations with the $\mathrm{CR}$ pressure terms in the conservative, Eulerian form for one-dimensional plane-parallel geometry (Kang et al. 2002; Kang \& Jones 2005, 2007),

$$
\frac{\partial \rho}{\partial t}+\frac{\partial(u \rho)}{\partial x}=0
$$

$$
\begin{gathered}
\frac{\partial(\rho u)}{\partial t}+\frac{\partial\left(\rho u^{2}+P_{g}+P_{c}\right)}{\partial x}=0, \\
\frac{\partial\left(\rho e_{g}\right)}{\partial t}+\frac{\partial}{\partial x}\left(\rho e_{g} u+P_{g} u\right)=-u \frac{\partial P_{c}}{\partial x}+W(x, t)-L(x, t),
\end{gathered}
$$

where $P_{g}$ and $P_{c}$ are the gas and CR pressures, respectively, $e_{g}=P_{g} /\left[\rho\left(\gamma_{g}-1\right)\right]+u^{2} / 2$ is the total gas energy per unit mass. The remaining variables, except for $L$ and $W$, have the usual meanings. The injection energy loss term, $L(x, t)$, accounts for the energy carried away by the suprathermal particles injected into the CR component at the subshock and is subtracted from the postshock gas immediately behind the subshock. The gas heating due to the Alfvén wave dissipation in the upstream region is represented by the term

$$
W(x, t)=-v_{A} \frac{\partial P_{c}}{\partial x},
$$

where $v_{A}=B / \sqrt{4 \pi \rho}$ is the local Alfvén speed (Paper I). These equations can be used to describe parallel shocks, where the large-scale magnetic field is aligned with the shock normal and the pressure contribution from the turbulent magnetic fields can be neglected.

The CR population is evolved by solving the diffusionconvection equation for the pitch angle-averaged distribution function, $f(x, p, t)$, in the form

$$
\frac{\partial g}{\partial t}+\left(u+u_{w}\right) \frac{\partial g}{\partial x}=\frac{1}{3} \frac{\partial}{\partial x}\left(u+u_{w}\right)\left(\frac{\partial g}{\partial y}-4 g\right)+\frac{\partial}{\partial x}\left[\kappa(x, y) \frac{\partial g}{\partial x}\right],
$$

where $g=p^{4} f$ and $y=\ln (p)$ (Skilling 1975a). Here, $\kappa(x, p)$ is the spatial diffusion coefficient. The CR population is isotropized with respect to the local Alfvénic wave turbulence, which would in general move at a speed $u_{w}$ with respect to the plasma. Since the Alfvén waves upstream of the subshock are expected to be established by the streaming instability, the wave speed is set there to be $u_{w}=v_{A}$. Downstream, it is likely that the Alfvénic turbulence is nearly isotropic, so we use $u_{w}=0$ there.

We consider two models for CR diffusion: Bohm diffusion and power-law diffusion,

$$
\begin{aligned}
& \kappa_{B}=\kappa^{*}\left(\frac{\rho_{0}}{\rho}\right)^{v} \frac{p^{2}}{\sqrt{p^{2}+1}} \\
& \kappa_{p l}=\kappa^{*}\left(\frac{\rho_{0}}{\rho}\right)^{\nu} p^{\alpha},
\end{aligned}
$$

with $\alpha=0.5-1$. Hereafter, the momentum is expressed in units of $m_{p c}$, where $m_{p}$ is the proton mass and $c$ is the speed of light. So, $\kappa^{*}$ is a constant of dimensions of length squared over time. As in our previous studies, we consider diffusion both without and with a density dependence, $\rho_{0} / \rho$; that is, either $v=0$ or $v=1$. The latter case quenches the $\mathrm{CR}$ acoustic instability (Drury 1984) and approximately accounts for the compressive amplification of Alfvén waves. Since we do not follow explicitly the amplification of magnetic fields due to streaming CRs, we simply assume that the field strength scales with compression and so the diffusion coefficient scales inversely with density. Bohm-like diffusion is an idealization of what is expected in a dynamically evolving CR-modified shock. As discussed in Section 2.3 the diffusion coefficient, which results from resonant scattering with Alfvén waves, varies inversely with 
the intensity of the resonant waves. The wave intensity is expected to be amplified as the shock evolves from upstream, ambient values via the streaming instability. Bohm diffusion represents the simplest nonlinear limited model for that process. The very highest momentum CRs will encounter ambient wave intensities, so perhaps below levels implied by Bohm diffusion. The model assumes that the streaming instability quickly amplifies those waves to nonlinear levels (e.g., Skilling 1975b; Lucek \& Bell 2000). We label the quantities upstream of the shock precursor by the subscript " 0 ," those immediately upstream of the gas subshock by "1," and those downstream by " 2 ." So, $\rho_{0}$, for example, stands for the density of the upstream gas.

Equations (1)-(3) and (5) are simultaneously integrated by the Cosmic-Ray Acceleration SHock (CRASH) code. The detailed description of the CRASH code can be found in Kang et al. (2002) and Paper I. Three features of CRASH are important to our discussion below. First, CRASH applies an adaptive mesh refinement technique around the subshock. So the precursor structure is adequately resolved to couple the gas to the CRs of low momenta, whose diffusion lengths can be at least several orders of magnitude smaller that the precursor width. Second, CRASH uses a subgrid shock tracking; that is, the subshock position is followed accurately within a single cell on the finest mesh refinement level. Consequently, the effective numerical subshock thickness needed to compute the spatial derivatives in Equation (5) is always less than the single cell size of the finest grid. Third, we calculate the exact subshock speed at each time step to adjust the rest frame of the simulation, so that the subshock is kept inside the same grid cell throughout. These three features enable us to obtain good numerical convergence in our solutions with a minimum of computational efforts. As shown in Paper I, the CRASH code can obtain reasonably converged dynamical solutions even when the grid spacing in the finest refined level is greater than the diffusion length of the lowest energy particles (i.e., $\left.\Delta x_{8}>l_{d}\left(p_{\text {inj }}\right)\right)$. This feature allows us to follow the particle acceleration for a large dynamic rage of $p_{\max } / p_{\text {inj }}$, typically, $\sim 10^{9}$, although the evolution of the energy spectrum at low energies and the early dynamical evolution of the shock structure may not be calculated accurately.

\subsection{Simulation Setup}

The injection and acceleration of CRs at shocks depend in general upon various shock parameters such as the Mach number, the magnetic field strength and obliquity angle, and the strength of the Alfvén turbulence responsible for scattering. In this study we focus on the relatively simple case of CR proton acceleration at quasi-parallel shocks, which is appropriately described by Equations (1)-(3). The details of simulation setup can be found in Paper I, and only a few essential features are briefly summarized here. Except for diffusion details, the setup described here is identical to those reported in Paper I.

As in Paper I, a shock is specified by the upstream gas temperature $T_{0}$ and the initial Mach number $M_{0}$. Two values of $T_{0}, 10^{4}$ and $10^{6} \mathrm{~K}$, are considered, representing the warm photoionized gas and the hot shock-heated gas often found in astrophysical environments, respectively. Then the initial shock speed is given as

$$
u_{s, i}=c_{s, 0} M_{0}=15 \mathrm{~km} \mathrm{~s}^{-1}\left(\frac{T_{0}}{10^{4}}\right)^{1 / 2} M_{0}
$$

where $c_{s, 0}$ is the sound speed of the upstream gas. All the simulations reported in this paper have $M_{0}=10$, which is large enough to produce significant CR modification. In Paper I, we considered a wide range of shock Mach numbers and examined the Mach-number dependence of the evolution of CR-modified shocks. The CR injection and acceleration efficiencies are determined mainly by the sonic Mach number and the relative Alfvén Mach number for shocks with $M_{0} \gtrsim 10$ (Kang et al. 2002; Kang 2003). On the other hand, they depend sensitively on other model parameters for shocks with lower Mach numbers. In this paper, we thus focus on the evolution of the CR spectrum at moderately strong shocks with $M_{0} \gtrsim 10$. We will consider the more complicated problem of weaker shocks in a separate paper.

In our problem, three normalization units are required for length, time, and mass. While ordinary, one-dimensional, ideal gasdynamic problems do not contain any intrinsic scales, the diffusion in the DSA problem introduces one; that is, either a diffusion length or a diffusion time, which of course depend on the particle momentum. So let $p^{\dagger}$ be a specific value of the highest momentum that we aim to achieve by the termination time of our simulations. Then the greatest width of the precursor is set by the diffusion length of the particles with $p^{\dagger}, l_{d}\left(p^{\dagger}\right)=\kappa\left(\rho_{0}, p^{\dagger}\right) / u_{s}$, while the time required for the precursor to reach that width is given by $t_{\text {acc }}\left(p^{\dagger}\right) \propto l_{d}\left(p^{\dagger}\right) / u_{s}$ (see Equation (9)). Hence we choose diffusion length and time for $p^{\dagger}, \hat{x}=\hat{\kappa} / \hat{u}$ and $\hat{t}=\hat{\kappa} / \hat{u}^{2}$, with $\hat{u}=u_{s, i}$ and $\hat{\kappa}=\kappa\left(\rho_{0}, p^{\dagger}\right)$, as the normalization units for length and time. For the normalization units for mass, we choose $\hat{\rho}=\rho_{0}$. Then the normalized quantities become $\tilde{x}=x / \hat{x}, \tilde{t}=t / \hat{t}$, $\tilde{u}=u / \hat{u}, \tilde{\kappa}=\kappa / \hat{\kappa}$, and $\tilde{\rho}=\rho / \hat{\rho}$. In addition, the normalized pressure is expressed as $\tilde{P}=P /\left(\hat{\rho} \hat{u}^{2}\right)$. With these choices, we expect that at time $\tilde{t} \sim 1$, the precursor width would be $\tilde{x} \sim \tilde{l}_{d}\left(p^{\dagger}\right) \sim 1$, for example. It should be clear that the physical contents of our normalization are ultimately determined by the value of $p^{\dagger}$ anticipated to correspond to $\tilde{t} \sim 1$ as well as by the form of $\kappa(\rho, p)$. In the simulations reported here, $p^{\dagger}$ was selected to give us the maximum span of $p$ that is consistent with our ability to obtain converged results with available computational resources. Our choice of $p^{\dagger}$ is especially dependent on the nonrelativistic momentum dependence of $\kappa(p)$. In particular, when the dependence is steep, $\kappa\left(p_{\text {inj }}\right)$ and $l_{d}\left(p_{\text {inj }}\right)$ can become extremely small compared with their relativistic values, necessitating very fine spatial resolution around the subshock.

In Table 1 , we list our numerical models classified by $T_{0}$ and $\kappa$. For example, T6P1 model adopts $T_{0}=10^{6} \mathrm{~K}$ and $\kappa_{p l}$ with $\alpha=1$ and $\nu=0$, while T4Bd model adopts $T=$ $10^{4} \mathrm{~K}$ and Bohm diffusion, $\kappa_{\mathrm{B}}$, with $v=1$. In the power-law diffusion models of T6P1 and T6P1d, $p^{\dagger} \sim 10^{6}$ is chosen for the normalization, so that $\tilde{\kappa}(\tilde{\rho}=1)=\tilde{\kappa}^{*} p=10^{-6} p$. For the Bohm diffusion models, T6Bd and T4Bd, on the other hand, $p^{\dagger} \sim 10^{2}$ is chosen, because the steep nonrelativistic form of the diffusion makes those models too costly for us to follow evolution to much higher CR momenta.

A specific example can clarify the application of these simulations to real situations. Let us consider a shock with $u_{s, i}=1.5 \times 10^{3} \mathrm{~km} \mathrm{~s}^{-1}$ propagating into the interstellar medium with $B=5 \mu \mathrm{G}$. Then in the Bohm limit that the relativistic CR scattering length equals the gyroradius, $\kappa^{*}=$ $m_{p} c^{2} /(3 e B)=6.3 \times 10^{21} \mathrm{~cm}^{2} \mathrm{~s}^{-1}$. For the T6P1 model, for instance, the normalization constants are $\hat{u}=1.5 \times 10^{3} \mathrm{~km} \mathrm{~s}^{-1}$ and $\hat{\kappa}=6.3 \times 10^{27} \mathrm{~cm}^{2} \mathrm{~s}^{-1}$, so $\hat{x}=4.2 \times 10^{19} \mathrm{~cm}$ and $\hat{t}=2.8 \times 10^{11} \mathrm{~s}$. 
Table 1

Preshock Temperature and Diffusion Coefficient in Numerical Models

\begin{tabular}{lccc}
\hline \hline Model Name & $T_{0}(\mathrm{~K})$ & $\kappa / \hat{\kappa}$ & Description for Diffusion Coefficient \\
\hline T6P1d & $10^{6}$ & $10^{-6} p\left(\rho_{0} / \rho\right)$ & Power-law diffusion with $\rho^{-1}$ dependence \\
T6P3/4d & $10^{6}$ & $10^{-6} p^{3 / 4}\left(\rho_{0} / \rho\right)$ & Power-law diffusion with $\rho^{-1}$ dependence \\
T6P1 & $10^{6}$ & $10^{-6} p$ & Power-law diffusion \\
T6P1/2 & $10^{6}$ & $1.78 \times 10^{-4} p^{1 / 2}$ & Power-law diffusion \\
T4P1d & $10^{4}$ & $10^{-5} p\left(\rho_{0} / \rho\right)$ & Power-law diffusion with $\rho^{-1}$ dependence \\
T6Bd & $10^{6}$ & $10^{-2} p^{2} / \sqrt{p^{2}+1}\left(\rho_{0} / \rho\right)$ & Bohm diffusion with $\rho^{-1}$ dependence \\
T4Bd & $10^{4}$ & $10^{-2} p^{2} / \sqrt{p^{2}+1}\left(\rho_{0} / \rho\right)$ & Bohm diffusion with $\rho^{-1}$ dependence \\
\hline
\end{tabular}

On the other hand, the time evolution of these shocks becomes approximately self-similar, as we will demonstrate. In that case the normalization choices above are entirely for the convenience of computation. We will eventually replace even these normalized physical variables with dimensionless similarity variables. To simplify the notation in the meantime, we hereafter drop the tilde from the normalized quantities as defined above.

Our simulations start with a purely gasdynamic shock of $M_{0}=10$ at rest at $x=0$, initialized according to RankineHugoniot relations with $u_{0}=-1, \rho_{0}=1$ and a gas adiabatic index, $\gamma_{g}=5 / 3$. So the initial shock speed is $u_{s, i}=1 \mathrm{in}$ code units. There are no pre-existing CRs, i.e., $P_{c}(x)=0$ at $t=0$.

\subsection{Thermal Leakage and Alfvén Wave Transport}

Although the shock Mach number is the key parameter that determines the evolution of CR-modified shocks, the thermal leakage injection and the Alfvén wave transport are important elements of DSA. They were discussed in detail in previous papers including Paper I. So here we briefly describe only the central concepts to make this paper self-contained.

In the CRASH code, the injection of suprathermal particles via thermal leakage is emulated numerically by adopting a "transparency function," $\tau_{\mathrm{esc}}\left(\epsilon_{B}, v\right)$, which expresses the probability of downstream particles at given random velocity, $v$, successfully swimming upstream across the subshock through the postshock MHD waves (Kang et al. 2002), whose amplitude is parameterized by $\epsilon_{B}$. Once such particles cross into the upstream flow, they are subject to scattering by the upstream Alfvén wave field, so participate in DSA. The condition that nonzero probability for suprathermal downstream particles to cross the subshock (i.e., $\tau_{\text {esc }}>0$ for $p>p_{\text {inj }}$ ) effectively selects the lowest momentum of the particles entering the CR population. The velocity $v$ obviously must exceed the flow speed of the downstream plasma, $u_{2}$. In addition, leaking particles must swim against the effective pondermotive force of MHD turbulence in the downstream plasma. The parameter, $\epsilon_{B}=B_{0} / B_{\perp}$ used to represent this, is the ratio of the magnitude of the largescale magnetic field aligned with the shock normal, $B_{0}$, to the amplitude of the postshock wave field that interacts with low energy particles, $B_{\perp}$. It is more difficult for particles to swim upstream when the wave turbulence is strong ( $\epsilon_{B}$ is small), leading to smaller injection rates. Malkov \& Völk (1998) argued on plasma physics grounds that it should be $0.25 \lesssim \epsilon_{B} \lesssim 0.35$. Our own CR shock simulations established that $\epsilon_{B} \sim 0.2-0.25$ leads to injection fractions in the range $\sim 10^{-4}$ to $10^{-3}$, which are similar to the commonly adopted values in other models (e.g., Malkov 1997; Amato \& Blasi 2005). In this study, we use $\epsilon_{B}=0.2$ for numerical models, although the choice is not critical to our conclusions.
The CR transport in DSA is controlled by the intensity, spectrum, and isotropy of the Alfvénic turbulence resonant with CRs. Upstream of the subshock, the Alfvénic turbulence is thought to be excited by the streaming CRs (e.g., Bell 1978; Lucek \& Bell 2000). Recently there has been much emphasis on the possible amplification of the large-scale magnetic field via nonresonant wave-particle interactions within the shock precursor (e.g., Bell 2004; Amato \& Blasi 2006; Vladmiriov et al. 2006). Those details will not concern us here; we make the simplifying assumption that the Alfvénic turbulence saturates and that scattering isotropizes the CR distribution in the frame moving with the mean Alfvén wave motion (see Equation (5)). Since the upstream waves are amplified by the CRs escaping upstream, the wave frame propagates in the upstream direction; i.e., $u_{w}>0$. Downstream, various processes should isotropize the Alfvén waves (e.g., Achterberg \& Blandford 1986), so the wave frame and the bulk flow frame coincide; i.e., $u_{w}=0$. This transition in $u_{w}$ across the subshock reduces the velocity jump experienced by CRs during DSA. Since it is really the velocity jump rather than the density jump that sets the momentum boost, this reduces the acceleration rate somewhat when the ratio of the upstream sound speed to the Alfvén speed is finite. An additional effect that has important impact is dissipation of Alfvén turbulence stimulated by the streaming CRs. That energy heats the inflowing plasma beyond adiabatic compression. The detailed physics is complicated and nonlinear, but we adopt the common, simple assumption that the dissipation is local and that the wave growth saturates, so that the dissipation rate matches the rate of wave stimulation (see Equation (4); Jones 1993; Berezhko \& Völk 1997). This energy deposition increases the sound speed of the precursor gas, thus reducing the Mach number of the flow into the subshock, again weakening DSA to some degree (e.g., Achterberg 1982). Thus, the CR acceleration becomes less efficient, when the Alfvén wave drift and heating terms are included (Berezhko \& Völk 1997; Kang \& Jones 2006).

The significance of these effects can be parameterized by the ratio of the magnetic field to thermal energy densities, $\theta=E_{B, 0} / E_{\mathrm{th}, 0}$, in the upstream region, which scales as the square of the ratio of the upstream Alfvén $\left(v_{A}\right)$ and sound speeds. In Paper I, we considered $0.1 \leqslant \theta \leqslant 1$; here we set $\theta=0.1$. The dependence of shock behaviors on that parameter are outlined in Paper I. The $\theta$ parameter can be related to the more commonly used shock Alfvénic Mach number, $M_{A, 0}=u_{s, i} / v_{A, 0}$, and the initial sonic Mach number, $M_{0}$, as $M_{A, 0}=M_{0} \sqrt{\gamma_{g}\left(\gamma_{g}-1\right) /(2 \theta)}$, where $v_{A, 0}=B_{0} / \sqrt{4 \pi \rho_{0}}$. With $\gamma_{g}=5 / 3$ and $\theta=0.1$, this translates into $M_{A, 0}=2.36 M_{0}$. So, for our $M_{0}=10$ shocks, $M_{A, 0} \approx 24$. Our initial shock speeds are $u_{s, i}=150 \mathrm{~km} \mathrm{~s}^{-1}$ for $T_{0}=10^{4} \mathrm{~K}$ and $u_{s, i}=1500 \mathrm{~km} \mathrm{~s}^{-1}$ for $T_{0}=10^{6} \mathrm{~K}$, corresponding, then, to $v_{A}=6.4 \mathrm{~km} \mathrm{~s}^{-1}$ 

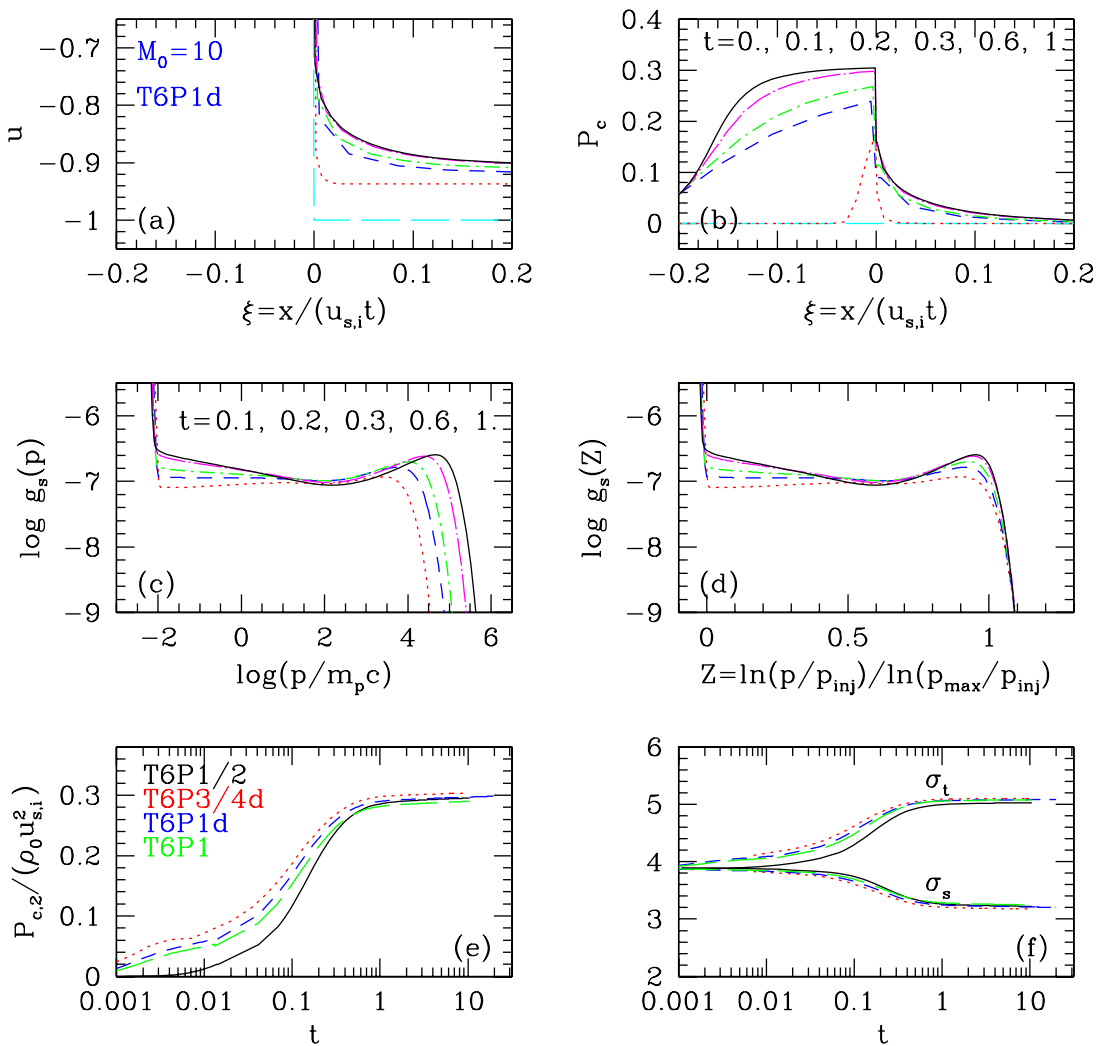

Figure 1. $(a)-(d)$ Snapshots of $M_{0}=10$ shock of T6P1d model up to $t=1$ in terms of a similarity variable $\xi \equiv x /\left(u_{s, i} t\right)$. The flow velocity, CR pressure, and CR distribution function at the subshock, $g_{s}(p)=f_{s}(p) p^{4}$, and $g_{s}(Z)$, are shown at $t=0.1$ (dotted lines), 0.2 (dashed), 0.3 (dot-dashed), 0.6 (dot-long dashed), and 1.0 (solid). The long dashed lines show the initial shock structure. $(e)$ and $(f)$ Time evolution of the postshock $\mathrm{CR}$ pressure and the compression ratios for $M_{0}=10$ shocks with four different models of diffusion coefficient $\kappa(p)$ (see Table 1).

(A color version of this figure is available in the online journal.)

and $v_{A}=64 \mathrm{~km} \mathrm{~s}^{-1}$, respectively. For our example magnetic field, $B_{0}=5 \mu \mathrm{G}$, the associated upstream gas density would be $\rho_{0} \approx 5 \times 10^{-24} \mathrm{~g} \mathrm{~cm}^{-3}$ and $\rho_{0} \approx 5 \times 10^{-26} \mathrm{~g} \mathrm{~cm}^{-3}$, respectively.

\section{RESULTS}

\subsection{Evolution Toward an Asymptotic State}

In the early evolutionary stage, as CRs are first injected and accelerated at the subshock, upstream diffusion creates a CR pressure gradient that decelerates and compresses the inflowing gas within a shock precursor. This leads to a gradual decrease of shock speed with respect to the upstream gas (Figures 1(a) and 1(b)). As the subshock consequently weakens, the CR injection rate decreases due to a reduced velocity jump across the subshock. The CR spectrum near $p_{\text {inj }}$ also steepens (Figures 1(c) and 1(d)). The total compression across the entire shock structure actually increases to about 5 in the Mach 10 shocks reported here. The highest momentum CRs respond to the total shock transition, which flattens the spectrum at higher momenta; i.e., the $\mathrm{CR}$ spectrum evolves the well known concave curvature between the lowest and the highest momenta. Each of these evolutionary features continue to be enhanced until preshock compression, CR injection at the subshock, and CR acceleration through the entire shock structure all reach selfconsistent dynamical equilibrium states (Figures 1(e) and 1(f)). Once compression in the precursor reaches the level at which DSA begins to saturate, meaning the reduced subshock strength reduces $\mathrm{CR}$ injection to maintain an equilibrium, the shock compression $\left(\sigma_{s}=\rho_{2} / \rho_{1}\right.$ and $\left.\sigma_{t}=\rho_{2} / \rho_{0}\right)$ as well as the gas and CR pressures should remain approximately constant during subsequent shock evolution. From that time on the structure of the precursor and the CR spectrum must evolve in tandem to maintain these dynamical features.

The CR pressure is calculated from the particle distribution function by

$$
P_{c}=\frac{4 \pi}{3} m_{p} c^{2} \int_{p_{\mathrm{inj}}}^{\infty} g(p) \frac{p}{\sqrt{p^{2}+1}} \frac{d p}{p} .
$$

To see how $P_{c}$ evolves during the early, nonrelativistic stage, consider the idealized the test-particle case where the CR distribution has a power-law form, $g(p)=g_{0}\left(p / p_{\text {inj }}\right)^{-\delta}$ up to $p=p_{\max }$, where $0<\delta \equiv\left(4-\sigma_{s}\right) /\left(\sigma_{s}-1\right)<0.5$ for the shock compression ratio of $4>\sigma_{s}>3$. Then one can roughly express $P_{c} \propto\left[\left(p_{\max } / p_{\text {inj }}\right)^{1-\delta}-1\right] \propto\left(p_{\max } / p_{\text {inj }}\right)^{1-\delta}$ for $p_{\text {inj }} \ll p_{\max }<1$. In a strong, unmodified shock, $1-\delta \approx 1$, and $P_{c}$ initially increases quickly as $P_{c} \propto p_{\max } / p_{\text {inj. }}$. We will show in Section 3.3, as the shock becomes modified toward the dynamical equilibrium state, that the CR pressure is dominated by relativistic particles and the $\mathrm{CR}$ spectrum evolves in a manner that leads to nearly constant postshock $P_{c, 2}$. These features in the evolution of $P_{c, 2}$ are illustrated in Figures 1(e) and 1(f). The time-asymptotic states are slightly different among different models, because the numerically realized $\mathrm{CR}$ injection rate depends weakly on $\kappa(p)$.

The mean acceleration time for a particle to reach $p_{\max }$ from $p_{\text {inj }}$ in the test-particle limit of DSA theory is given by (e.g., 

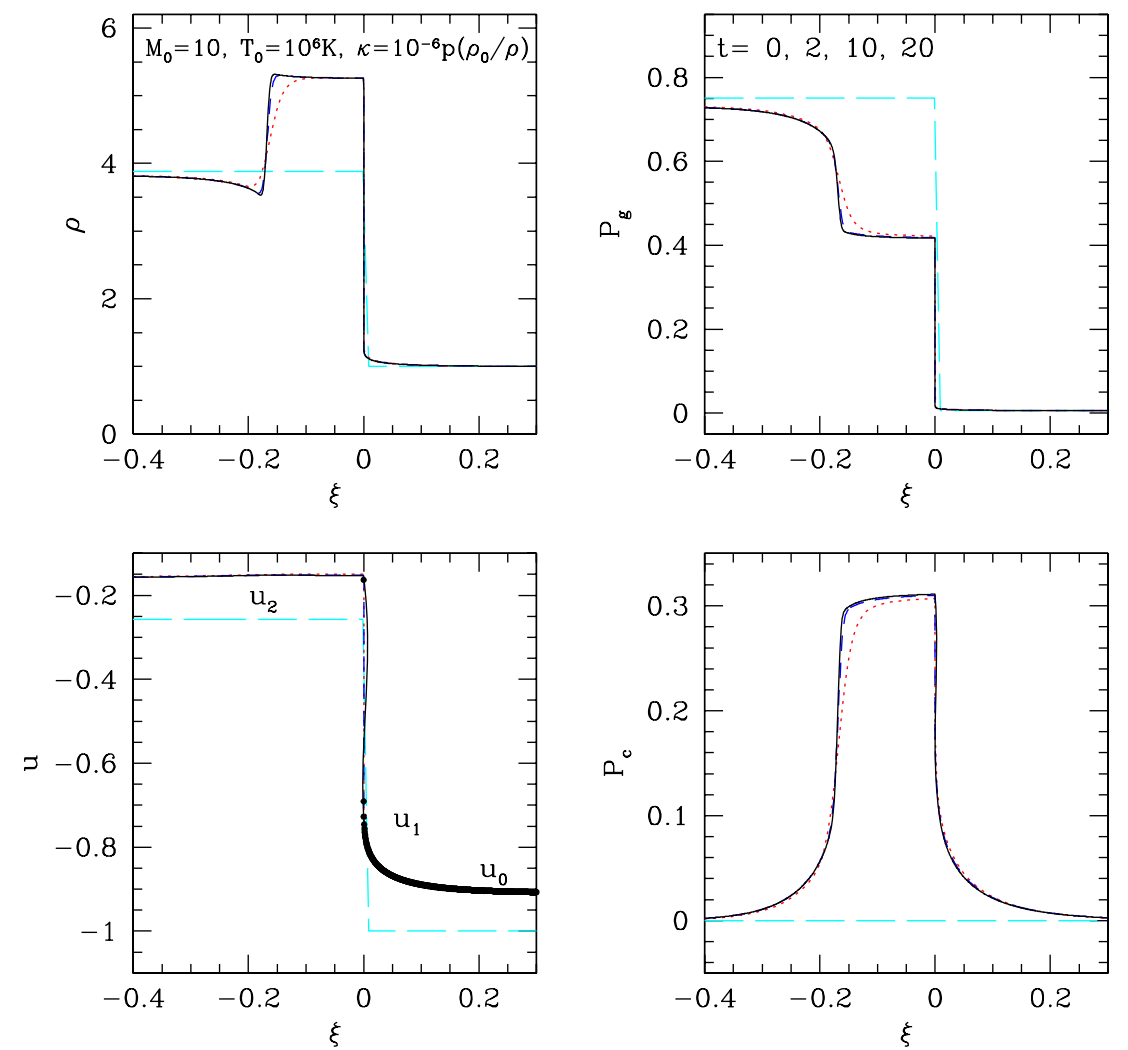

Figure 2. Self-similar evolution of $M_{0}=10$ shock of T6P1d model. The shock structure is shown at $t=2$ (dotted lines), 10 (dashed), and 20 (solid) as a function of the similarity variable $\xi=x /\left(u_{s, i} t\right)$ in the shock rest frame. The long dashed lines show the initial shock structure.

(A color version of this figure is available in the online journal.)

Drury 1983)

$$
t_{\mathrm{acc}}=\frac{3}{u_{0}-u_{2}} \int_{p_{\mathrm{inj}}}^{p_{\max }}\left(\frac{\kappa_{0}}{u_{0}}+\frac{\kappa_{2}}{u_{2}}\right) \frac{d p}{p} .
$$

For power-law diffusion with density dependence, $\kappa_{p l}=$ $\kappa_{*} p^{\alpha}\left(\rho_{0} / \rho\right)^{\nu}$, the maximum momentum can be estimated by setting $t=t_{\text {acc }}$ as

$$
p_{\max }(t) \approx\left[\frac{\alpha\left(\sigma_{t}-1\right)}{3 \sigma_{t}\left(1+\sigma_{t}^{1-\nu}\right)} \frac{u_{s}^{2}}{\kappa^{*}} t\right]^{1 / \alpha}=\left[f_{c} \frac{u_{s}^{2}}{\kappa^{*}} t\right]^{1 / \alpha},
$$

where $f_{c} \equiv \alpha\left(\sigma_{t}-1\right) /\left[3 \sigma_{t}\left(1+\sigma_{t}^{1-\nu}\right)\right]$ is a constant factor during the self-similar stage and $u_{s}$ is the shock speed in the timeasymptotic limit. As the feedback from CRs becomes important, the shock speed relative to far upstream flow is reduced, typically about 10\%-20\% for the shock parameters considered here (i.e., $u_{s} \approx[0.8-0.9] u_{s, i}$. With $\alpha=1$ and $v=1$, for a typical value of $\sigma_{t} \approx 5.3$ for an $M_{0}=10$ shock, $f_{c} \approx 0.13$.

In an evolving CR shock, at a given shock age of $t$, the powerlaw spectrum should extend roughly to $p_{\max }(t)$ above which it should decrease exponentially. Then the diffusion length of the most energetic particles increases linearly with time as

$$
l_{\max }(t) \equiv \frac{\kappa^{*} p_{\max }^{\alpha}(t)}{u_{s}}=f_{c} u_{s} t
$$

So $l_{\max }(t)$ depends only on the characteristic length $u_{s} t$, independent of the size of the diffusion coefficient, although at a given time the particles are accelerated to higher energies with smaller values of $\kappa^{*}$. Since the precursor scale height is proportional to $l_{\max }$, the precursor broadens linearly with time, again independent of the size of $\kappa^{*}$. This is valid even for the Bohm diffusion if $p_{\max } \gg 1$, since $\kappa_{\mathrm{B}} \approx \kappa^{*} p$ for $p \gg 1$. Thus, the hydrodynamic structure of evolving CR shocks does not depend on the diffusion coefficient, even though the CR diffusion introduces the diffusion length and timescales in the problem.

\subsection{Shock Structure and CR Spectrum in Self-similar Stage}

After the precursor growth reaches a time-asymptotic form, the shock structure follows roughly the self-similar evolution and stretches linearly with time, as noted above. Thus, we show in Figure 2 the evolution of an $M_{0}=10$ shock with T6P1d model in terms of the similarity variable, $\xi=x /\left(u_{s, i} t\right)$, for $t>1$ (i.e., later stage of the shock shown in Figure 1). The time-asymptotic shock speed approaches $u_{s}=u_{0} \approx 0.9 u_{s, i}$ for these shock parameters. The reduction in shock speed results from the increase in $\sigma_{t}$, so depends upon the degree of shock modification. Here $\sigma_{t} \approx 5.3, \alpha=1, v=1$, so Equation (11) give $l_{\max } \approx 0.13 u_{s} t$, which corresponds to the precursor scale height in terms of $\xi, H_{\xi} \equiv l_{\max } /\left(u_{s, i} t\right) \approx 0.12$.

We also show the approximate self-similar evolution of the shock structure for four additional models with $\kappa(\rho, p)$ listed in Table 1 (Figure 3). As discussed in Section 3.1, the overall shock structure at a given time $t$ is roughly independent of the diffusion coefficient, except for some minor details in the shock profile that have developed in the early stage. Also the shock evolution seems to be approximately self-similar in all the models, as shown in the middle and right panels of Figure 3. Of course, with different values of $\kappa^{*}$ and $\alpha$, on the other hand, the highest 


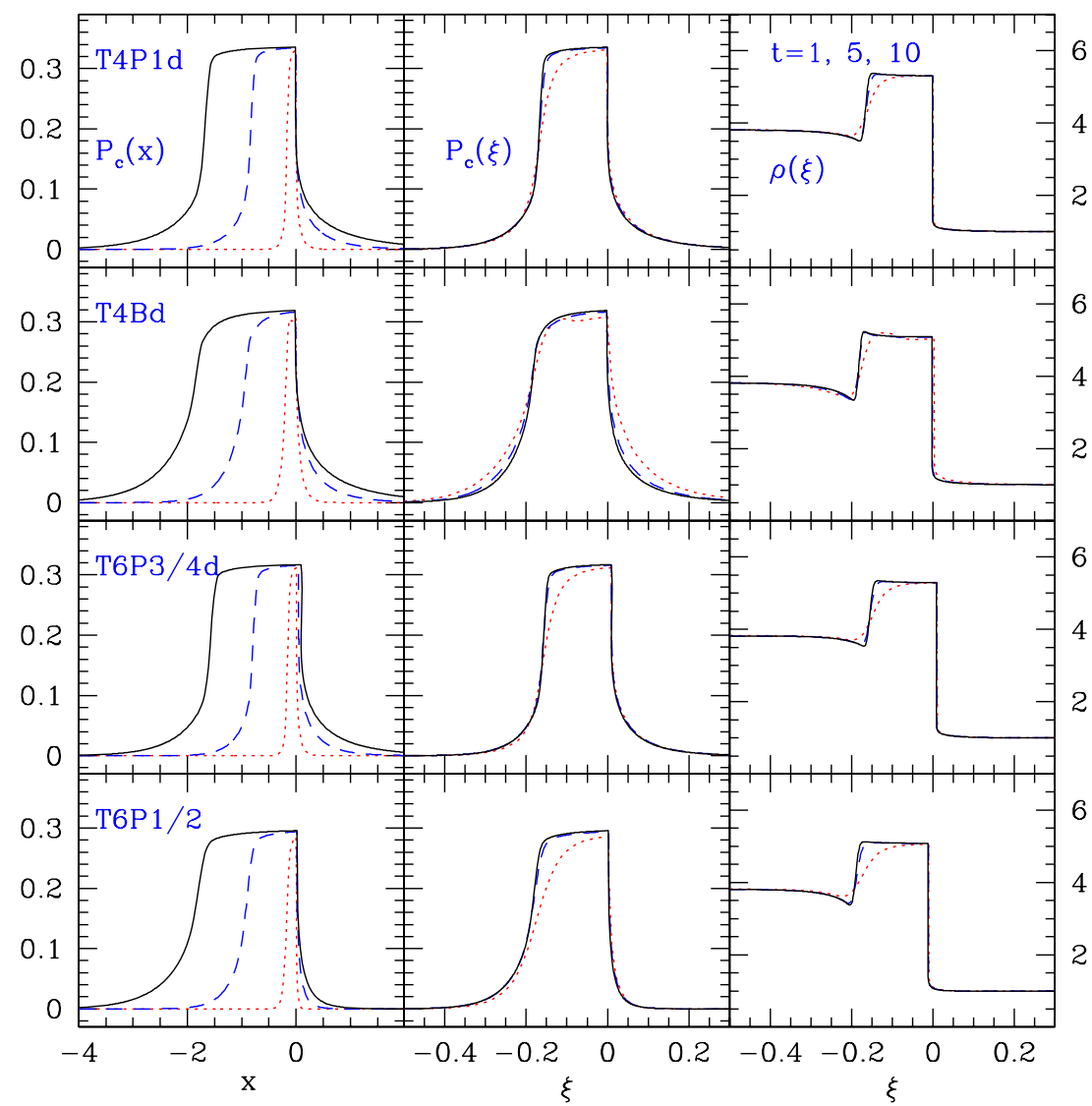

Figure 3. Self-similar evolution of $M_{0}=10$ shock of four different models listed in Table 1 , shown at $t=1$ (dotted lines), 5 (dashed), and 10 (solid). The CR pressure is shown as a function of $x$ (left panels) and the spatial similarity variable $\xi=x /\left(u_{s, i} t\right)$ (middle panels). The gas density is shown in the right panels.

(A color version of this figure is available in the online journal.)

momentum of the CR spectrum at a given time depends on $\kappa$ (see Figure 5).

Figures 4(a) and 4(b) shows how the particle distribution at the subshock, $g_{s}(p)=f\left(x_{s}, p\right) p^{4}$, evolves during the selfsimilar stage, extending to higher $p_{\max }$. For this model Equation (10) gives $p_{\max } \approx\left(0.1 / \kappa^{*}\right) t=10^{5} t$. This estimate is quite consistent with the evolution of $g_{s}(p)$ shown in this figure. The peak value of $g_{s}(p)$ near $p_{\max }$ seems to remain constant during the self-similar stage. This reflects the fact that $P_{c, 2}$ remains constant, as it must once DSA is saturated, and the fact that $P_{c}$ is dominated by relativistic CRs near $p_{\max }$ for strong shocks.

The injection momentum, $p_{\text {inj }} \propto \sqrt{P_{g, 2} / \rho_{2}}$, becomes constant in time after the initial adjustment, because the postshock state is fixed in the self-similar evolution stage. Then the value of $g_{s}\left(p_{\text {inj }}\right)$ is fixed by $g_{s, \text { th }}\left(p_{\text {inj }}\right)$, the thermal distribution of the postshock gas at $p_{\text {inj }}$, and stays constant, too.

Let us suppose particles with a given momentum $p_{1}$ experience on average the velocity jump over the diffusion length $\xi_{1}=l_{d}\left(p_{1}\right) /\left(u_{s} t_{1}\right), \Delta u\left(\xi_{1}\right)$, at time $t_{1}$. At a later time $t$ they will be accelerated to $p=p_{1} \cdot\left(t / t_{1}\right)^{1 / \alpha}$ and diffuse over the scale, $\xi=l_{d}(p) /\left(u_{s} t\right)=\xi_{1}$. So they experience the same velocity jump $\Delta u\left(\xi_{1}\right)$, as long as the velocity profile, $u(\xi)$, remains constant during the self-similar stage. Then the spectral slopes plotted in terms of $p / p_{\max }$ should retain a similar shape over time. The slope of the distribution function at the subshock, $q=-d \ln g_{s} / d \ln p+4$, and the slope of the volumeintegrated distribution function, $Q=-d \ln G / d \ln p+4$ (where $\left.G=\int g d x\right)$, as a function of $p / p_{\max }(t)$ are shown in Figure 4(d). Low energy particles near $p_{\text {inj }}$ experience the subshock compres- sion only, while highest momentum particles near $p_{\max }$ feel the total shock compression. So $q(p) \approx q_{s}=3 \sigma_{s} /\left(\sigma_{s}-1\right)$ for $p \sim p_{\text {inj }}$, while $q(p) \approx q_{t}=3 \sigma_{t} /\left(\sigma_{t}-1\right)$ for $p \sim p_{\max }$. The numerical results are roughly consistent with such expectations.

Consequently, to a good approximation, $g_{s}(p)$ evolves with fixed amplitudes, $g_{s}\left(p_{\text {inj }}\right)$ and $g_{s}\left(p_{\max }\right)$, and with fixed spectral slopes, $q_{s}$ and $q_{t}$ at $p_{\text {inj }}$ and $p_{\max }$, respectively, while stretching to higher $p_{\max }(t)$. The volume-integrated distribution function, $G(p)$, also displays a similar behavior as $g_{s}(p)$. In the bottom panels of Figure 4, $G(p) / t$ and $G(Z) / t$ are shown, noting that the kinetic energy passed through the shock front increases linearly with time.

In Paper I, based on the DSA simulation results for $t \leqslant 10$, we suggested that the distribution function may become selfsimilar in terms of the momentum similarity variable, $Z$, defined in Section 1. If we define the "partial pressure function" as

$$
F(Z) \equiv g(Z) \frac{p}{\sqrt{p^{2}+1}} \ln \left(\frac{p_{\text {max }}}{p_{\text {inj }}}\right)
$$

then the CR pressure is given by $P_{c} \propto \int_{0}^{\infty} F(Z) d Z$. We suggested there that the postshock CR pressure stays constant because the evolution of $F(Z)$ becomes self-similar. As can be seen in Figures 4(b) and 4(c), the functions $g_{s}(Z)$ and $F_{s}(Z)$ at the subshock seem to change very slowly, giving the false impression that $F_{s}(Z)$ might be self-similar in terms of the variable $Z$. However, the constant shape of $F(Z)$ cannot be compatible with the self-similar evolution of the precursor and shock profile. Since $f_{s}(p) \propto\left(p / p_{\text {inj }}\right)^{-q_{s}}$ at $Z \sim 0$ and 

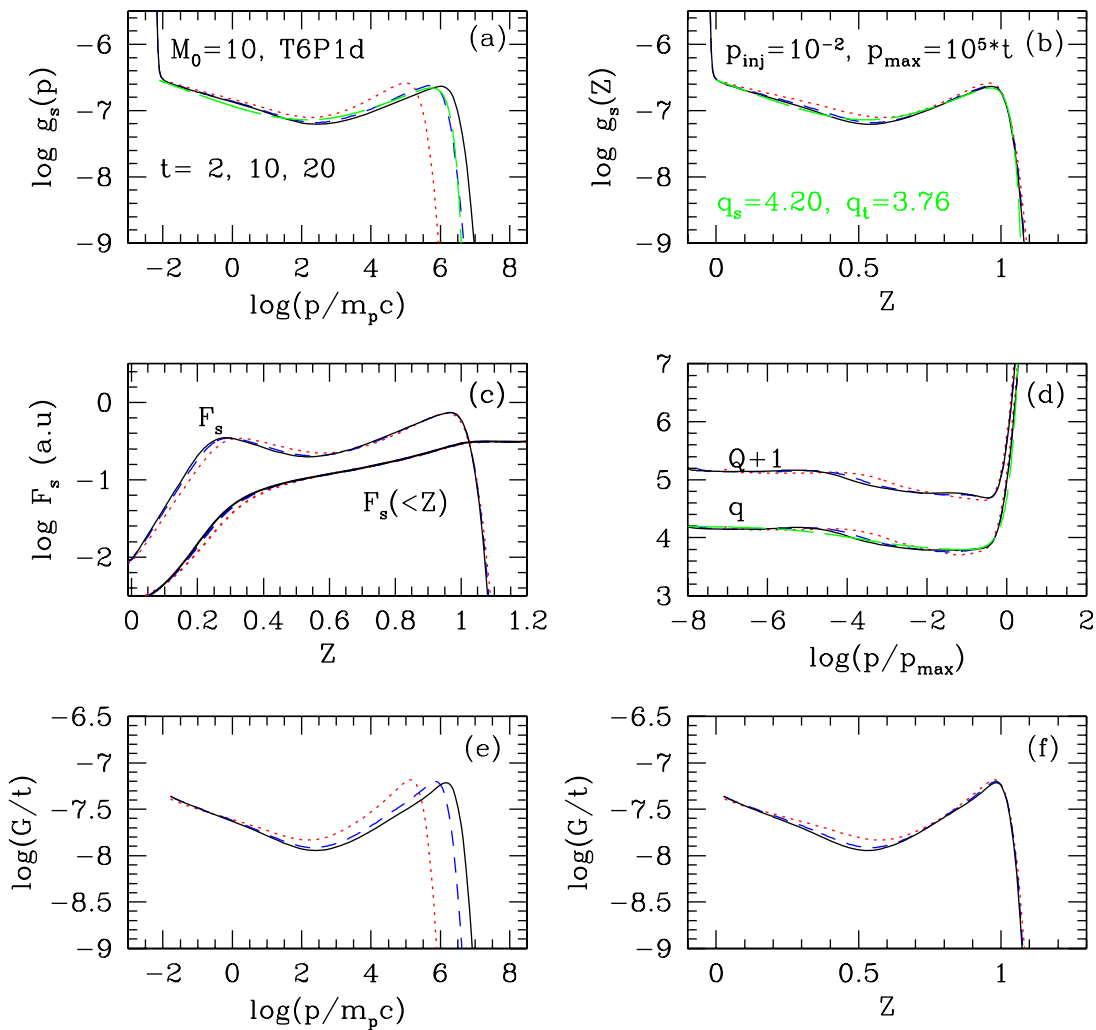

Figure 4. CR distribution function for $M_{0}=10$ shock of T6P1d model, as shown in Figure 2, at $t=2$ (dotted lines), 10 (dashed), and 20 (solid). (a) and (b) The distribution function at the subshock, $g_{s}(p)$ and $g_{s}(Z)$, where $Z=\ln \left(p / p_{\text {inj }}\right) / \ln \left(p_{\max } / p_{\text {inj }}\right)$. $(c)$ The partial pressure, $F_{s}$, defined in Equation $(12)$ and its cumulative distribution, $F_{s}(<z)$. (d) The power-law slopes, $q=-d \ln g_{s} / d \ln p+4$ and $Q=-d \ln G / d \ln p+4$. (e) and (f) The volume-integrated distribution function $G=\int g d x$ plotted against $\log (p)$ or $Z$. The long dashed lines in $(a),(b)$, and $(d)$ show the analytic fitting given in Equation (14) with $q_{s}=4.20, q_{t}=3.76$, $p_{\text {inj }}=10^{-2}$, and $p_{\text {max }}=10^{5} t$ at $t=10$.

(A color version of this figure is available in the online journal.)

$f_{s}(p) \propto\left(p / p_{\max }\right)^{-q_{t}}$ at $Z \sim 1$ with constant values of $p_{\text {inj }}$, $q_{s}$, and $q_{t}$, the shape of $F(Z)$ should evolve accordingly in the self-similar stage (see Figure 9 below).

Figure 5 shows how the evolution of $g_{s}(p)$ depends on the diffusion coefficient and preshock temperature, while other parameters, $M_{0}=10, \epsilon_{B}=0.2$, and $\theta=0.1$, are fixed. The same set of models is shown as in Figure 3. The shape of $g_{s}(p)$ is somewhat different among different models, although it seems to remain similar in time for a given model. The causes of such differences can be understood as follows. First of all, the value of $g_{s}\left(p_{\text {inj }}\right) \approx g_{s, \text { th }}\left(p_{\text {inj }}\right)$ depends on the value of $p_{\text {inj }} \propto\left(u_{s} / c\right) \propto$ $M_{0} \sqrt{T_{0}}$. Second, the numerically realized "effective" value of the injection momentum depends on the diffusion coefficient and grid spacing, leading to slightly different injection rates and shock structures. Thus, the postshock $P_{c, 2}$ and the compression ratios (i.e., the shock structure) depend weakly on diffusion coefficient, as shown in Figures 1(e) and 1(f). The ensuing CR spectra have slightly different values of $q_{s}$ and $q_{t}$ as shown in Figure 5.

The spectral slope of the CR spectrum is determined by the mean velocity jump that the particles experience across the shock structure. Here, we examine how the precursor velocity profile depends on the diffusion model. Figure 6(a) shows the velocity structure $U(\xi)=-u(\xi)$ in the precursor $(\xi>0)$ for five different diffusion models, where $u(\xi)$ is defined as shown in Figure 2. We use the velocity data in the finest level grid as well as in the base grid. The velocity profiles are quite similar in all the models except that the model with $\kappa \propto p^{1 / 2}$ shows a slightly different pattern at small scales $(\log \xi<-5)$.
Since the particles with momentum $p$ feel on average the velocity jump over the corresponding diffusion length, we can find the velocity $U\left(\xi_{p}\right)$ at the distance from the shock that satisfies $x=l_{d}(p)=\xi_{p} \cdot\left(u_{s, i} t\right)$. Using Equation (10), we find then $\xi_{p}=f_{c}\left(u_{s} / u_{s, i}\right)\left(p / p_{\max }\right)^{\alpha}$. Then the particles with the same ratio of $p / p_{\text {max }}$ diffuse over the same similarity scale, $\xi_{p}$, and feel the same velocity jump, $U\left(\xi_{p}\right)+u_{w}\left(\xi_{p}\right)-U_{2}$ across the shock. Thus, the spectral slope can be estimated from the velocity profile as (e.g., Berezhko \& Ellison 1999)

$$
q_{u}(p)=q_{u}\left(\xi_{p}\right)=\frac{3\left(U+u_{w}\right)}{U+u_{w}-U_{2}}+\frac{d \ln \left(U+u_{w}-U_{2}\right)}{d \ln p} .
$$

Figure 6(b) shows the spectral slope, $q_{u}$, which is calculated from numerical results of $U+u_{w}$ for different models. These curves compare to the $q(p)$ curves in Figure 5.

The numerical convergence issue should be discussed here. The base grid had a spatial resolution $\Delta x_{0}=2 \times 10^{-3}$ in the code units. The small region around the subshock was refined with a number of levels increasing to eight, giving there a spatial resolution $\Delta x_{8}=7.8 \times 10^{-6}$. This structure was sufficient to produce dynamically converged solutions as discussed in Paper I. The diffusion length near $p_{\text {inj }} \approx 10^{-2}$ is, for instance, $l_{d}\left(p_{\text {inj }}\right) \approx \kappa\left(p_{\text {inj }}\right) / u_{s, i} \approx 10^{-8}$ in T6P1d model and $\mathrm{1}_{d}\left(p_{\text {inj }}\right) \approx 2 \times 10^{-5}$ in T6P1/2 model, where all quantities are given in the code units. So the solution for Equation (5) is not resolved for the lowest energy particles in T6P1d model, while it should be well resolved in T6P1/2 model. Since low energy particles cannot see the flow structure shorter than the minimum 


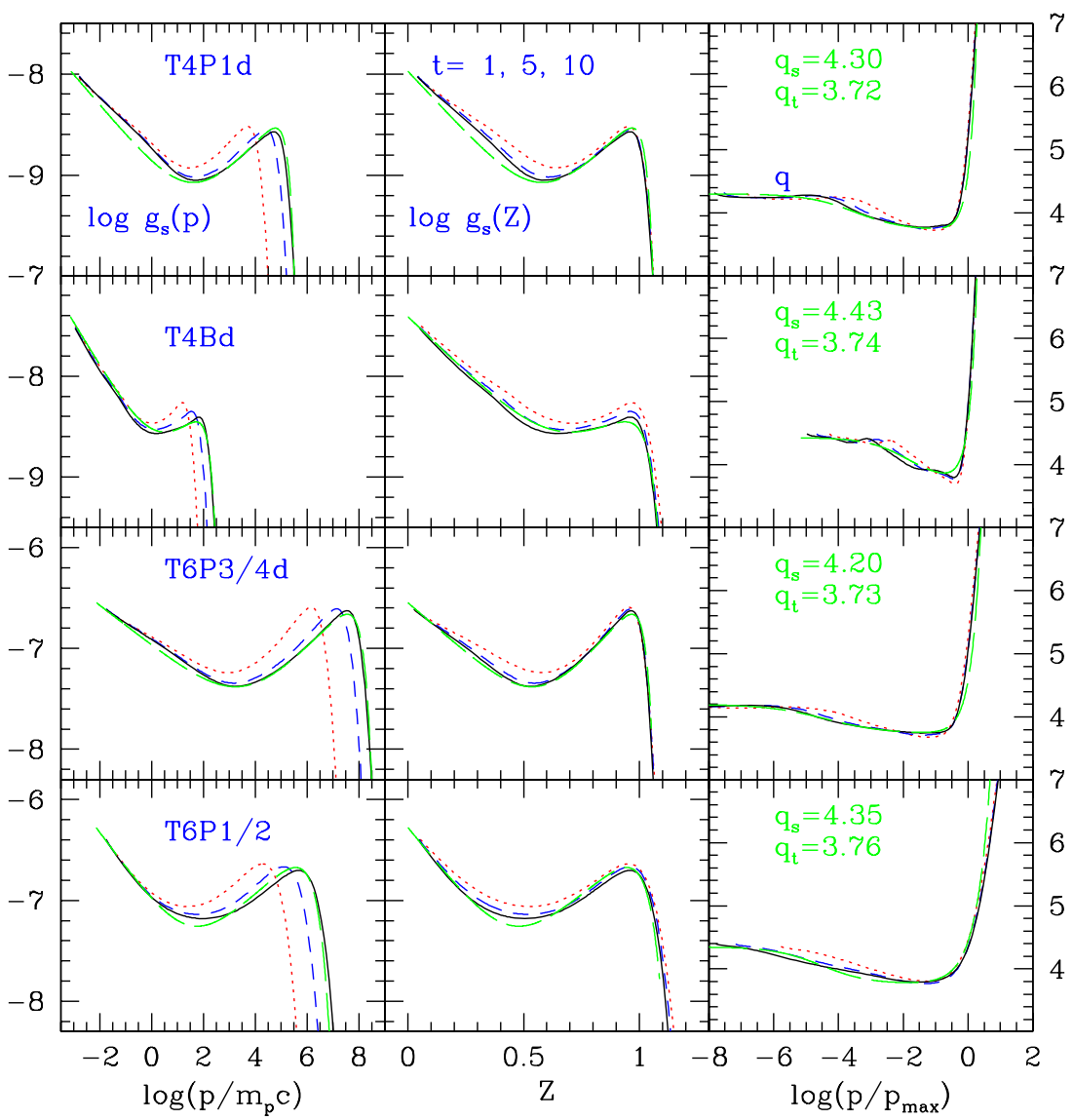

Figure 5. CR distribution function at the subshock, $g_{s}(p)$ and $g_{s}(Z)$, and the power-law slopes, $q=-d \ln g_{s} / d \ln p+4$ are shown at $t=1$ (dotted lines), 5 (dashed), and 10 (solid) for the four diffusion models shown in Figure 3. The long dashed lines show the analytic fitting given in Equation (14) at $t=10$. The adopted values of $q_{s}$ and $q_{t}$ are given for each model.

(A color version of this figure is available in the online journal.)
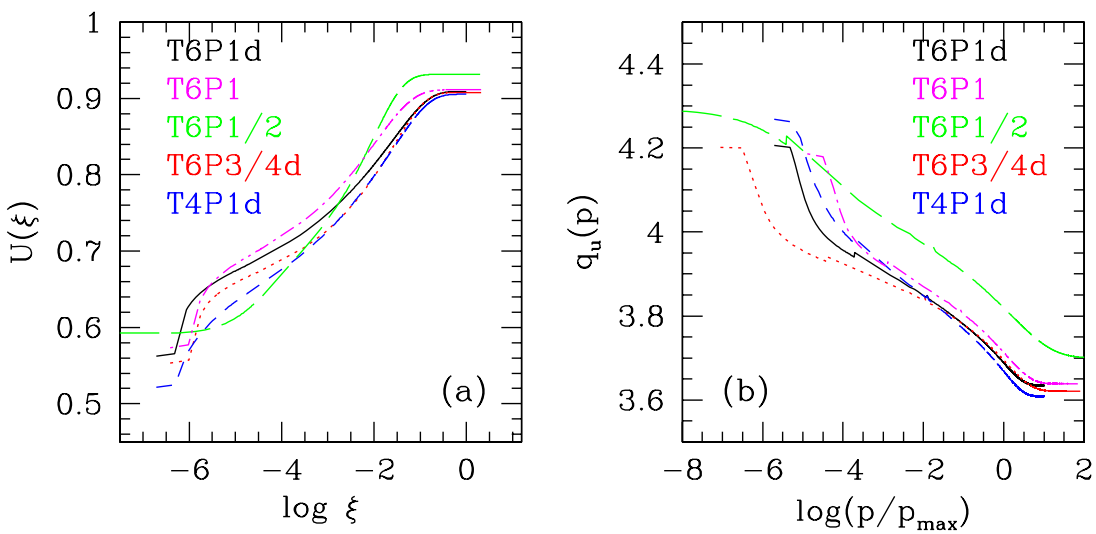

Figure 6. (a) Velocity profiles in the precursor as a function of the similarity distance from the subshock for five different models with $M_{0}=10$ listed in Table 1 . (b) The power slope calculated with Equation (13) using the velocity profile shown in (a).

(A color version of this figure is available in the online journal.)

numerical thickness of the subshock, i.e., $\Delta x_{8}$, corresponding to the effective diffusion length of $p \sim 10$ for T6P1d model, all particles below $p<10$ feel the same subshock compression, independent of their diffusion lengths. This leads to a more or less constant $q(p) \approx q_{s}$ for $p<10$. The models shown in Figures 4 and 5 exhibit this trend except T6P1/2 model in which the diffusion of the injected particles are well resolved with $\Delta x_{8} / l_{d}\left(p_{\text {inj }}\right)=0.4$.

The momentum integration of $g(x, p)$, i.e., the CR pressure, is self-similar in the spatial similarity variable $\xi$. Moreover, the CR distribution at the subshock, $g_{s}(Z)$, and the volume- integrated distribution, $G(Z)$, both change very slowly in time, when they are expressed in terms of $Z$. So we expect that the distribution function $g$ in the plane of $(\xi, Z)$ should change only secularly during the self-similar stage, although, as mentioned before, $g(Z)$ does not evolve self-similarly in the $Z$ space (Figure 7). The phase space distribution of $g(\xi, Z)$ shows that most of low energy particles $(Z<0.5)$ are confined within $-0.2 \lesssim \xi \lesssim 0.1$, while the highest energy particles $(Z \sim 1)$ diffuse over $-1 \leqslant \xi \leqslant 1$. Thus, far away from the subshock, both downstream and upstream, relativistic particles dominate the CR energy spectrum. 

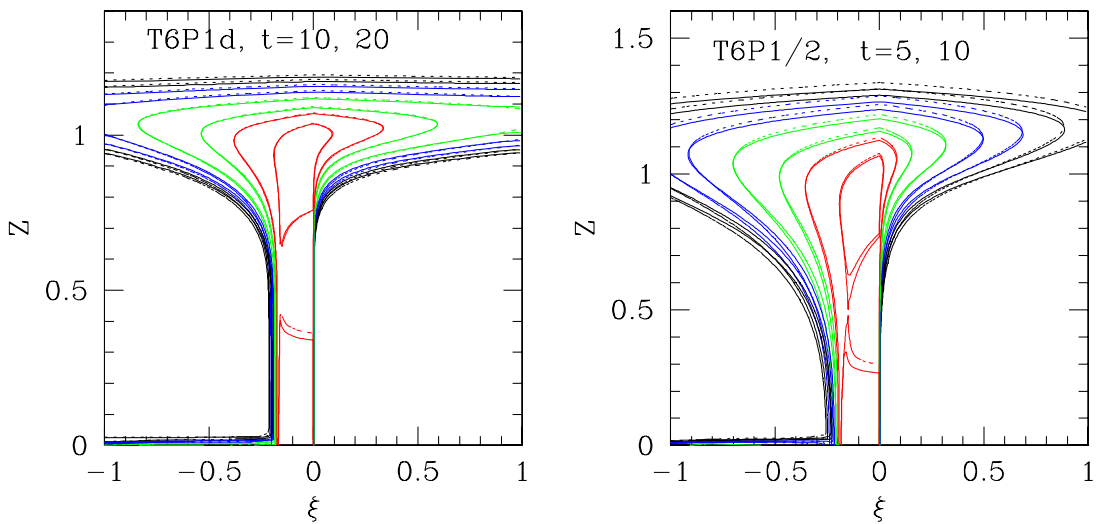

Figure 7. Left panel: contour plots of $g(\xi, Z)$ at $t=10$ (dotted lines), 20 (solid lines) for T6P1d model. Right panel: contour plots of $g(\xi, Z)$ at $t=5$ (dotted lines), 10 (solid lines) for T6P1/2 model.

(A color version of this figure is available in the online journal.)

\subsection{Analytic Approximation for CR Spectrum}

Based on the results of DSA simulations described in the previous subsections, we suggest that the $\mathrm{CR}$ spectrum at CR shocks with $M_{0} \gtrsim 10$ in the self-similar stage can be approximated by the sum of two power-law functions with an exponential cutoff as follows: for $p_{\max } \gg 1 \gg p_{\text {inj }}$,

$$
\begin{aligned}
g_{s}(p)= & {\left[g_{0} \cdot\left(\frac{p}{p_{\text {inj }}}\right)^{-q_{s}+4}+g_{1} \cdot\left(\frac{p}{p_{\max }}\right)^{-q_{t}+4}\right] } \\
& \times \exp \left[-\left(\frac{p}{1.5 p_{\max }}\right)^{2 \alpha}\right],
\end{aligned}
$$

where $q_{s}>4$ and $q_{t}<4$. The specific functional form of the exponential cutoff was found by fitting the numerical simulation results (see Figures 4 and 5). We have shown that, after the precursor has developed fully, the CR pressure at the subshock approaches a time-asymptotic value, which leads to the self-similar evolution of the entire shock structure. Then the parameters, $p_{\text {inj }}, q_{s}$, and $q_{t}$ as well as $g_{0} \approx g_{s, \text { th }}\left(p_{\text {inj }}\right)$, become constant in time. Also, the value of $g_{1}$ seems to stay roughly constant, according the simulation results. We will show below $g_{1}$ has to be approximately constant, if $P_{c, 2}$ remains constant during the self-similar stage. Then the only time-dependent parameter in Equation (14) is $p_{\max }(t)$, which can be estimated from Equation (10).

Now let us examine how $P_{c, 2}$ evolves in time with the proposed form of $g_{s}(p)$ as $p_{\max }$ increases to large values. Adopting $\alpha=1$, the contributions due to the low and high energy components can be calculated as

$$
\begin{aligned}
& P_{L} \equiv \int_{p_{\text {inj }}}^{p_{\max }} g_{0}\left(\frac{p}{p_{\text {inj }}}\right)^{-q_{s}+4} \exp \left[-\left(\frac{p}{1.5 p_{\text {max }}}\right)^{2}\right] \frac{p}{\sqrt{p^{2}+1}} \frac{d p}{p}, \\
& P_{H} \equiv \int_{p_{\text {inj }}}^{p_{\max }} g_{1}\left(\frac{p}{p_{\text {max }}}\right)^{-q_{t}+4} \exp \left[-\left(\frac{p}{1.5 p_{\text {max }}}\right)^{2}\right] \frac{p}{\sqrt{p^{2}+1}} \frac{d p}{p} .
\end{aligned}
$$

In Figure 8 , we show the values of $P_{L} / g_{0}$ and $P_{H} / g_{1}$ as a function of $p_{\max }$ for several values of $q_{s}$ and $q_{t}$ and $p_{\text {inj }}=10^{-2}$. In $M_{0}=10$ shocks the typical values of the compression ratios are $\sigma_{s} \approx 3.1$ and $\sigma_{t} \approx 5.0$, so $q_{s} \approx 4.4$ and $q_{t} \approx 3.75$. The plot shows that both $P_{L} / g_{0}$ and $P_{H} / g_{1}$ become constant as $p_{\max }$ becomes ultrarelativistic, if the shock flow is modified so that $\sigma_{s} \rightarrow 3$ and $\sigma_{t} \gg 4$. This explains why $P_{c, 2}$ approaches an asymptotic value as $p_{\max }$ becomes large, leading to the self-similar evolution stage, after the subshock weakens to the subshock Mach number, $M_{1} \sim 3-4$ and the total compression becomes greater than 4 . Therefore $g_{1}$ should stay constant, if $P_{c, 2}$ becomes constant in the self-similar stage.

The amplitude $g_{1}$ can be estimated, if, for example, $P_{c, 2}$ is known from the DSA simulations; i.e., the CR pressure obtained with the proposed analytic form of $g_{s}$ should be equal to the value of $P_{c, 2}$ from the DSA simulations. Alternatively, as outlined in the Appendix, empirical scaling relations established from simulations can connect $P_{c, 2}$ through simple physics to basic shock parameters. Then all the parameters necessary to construct approximations to the $\mathrm{CR}$ distribution function as given in Equation (14) at arbitrary time $t$ are known for the self-similar evolution stage. Since the time-asymptotic, selfsimilar solution of evolving CR shocks cannot be found (semi-) analytically either from the conservation equations or from the boundary conditions, we have to rely at least in part on numerical simulations to estimate the parameters $p_{\text {inj }}, g_{0}, \sigma_{s}, \sigma_{t}$, and $P_{c, 2}$ for given shock parameters. The analytic fitting forms that can approximate the DSA simulation results are described in the Appendix.

In Figures 4 and 5, we compare the analytic fitting formula in Equation (14) with the results of our DSA simulations. They show good agreement. These plots also demonstrate that $g_{s}\left(p_{\max }\right)$, and therefore, $g_{1}$, remains constant in the self-similar evolution stage. The compression ratios shown in Figure 1 are $\sigma_{s} \approx 3.2$ and $\sigma_{t} \approx 5.0$, so the power-law indices calculated with these ratios are $q_{s}=4.36$ and $q_{t}=3.75$. But the numerical value of $q=-d \ln f_{s} / d \ln p$ near $p_{\text {inj }}$ is 4.2 , because the diffusion of low energy particles is not resolved fully. The minimum value of $q=-d \ln f_{s} / d \ln p$ near $p_{\max }$ is 3.79 , slightly larger than $q_{t}$, because of the exponential cutoff. Just to demonstrate how the proposed form of $g_{s}(p)$ fits the simulation results, we use $q_{s}=4.2$ and $q_{t}=3.76$ instead for the curve shown in Figure 4. We note that Berezhko \& Ellison (1999) suggested the minimum value of $q$ is $q_{\min }=$ $3.5+\left(3.5-0.5 \sigma_{s}\right) /\left(2 \sigma_{t}-\sigma_{s}-1\right)$. With our compression ratios, $\sigma_{s}=3.2$ and $\sigma_{t}=5.0$, this gives $q_{\min }=3.83$, which is slightly larger than our estimate of 3.79 .

Using Equations (10) and (14), we can estimate the CR spectrum $g_{s}$ at arbitrary time in the self-similar stage, as demonstrated in Figure 9. Here the value of $g_{1}$ is fixed by setting $P_{c, 2}=0.30$ at $t=1$ and then the same value of $g_{1}$ is used for the time $t>10$. From the curves of cumulative $F_{s}(<Z)$, we 

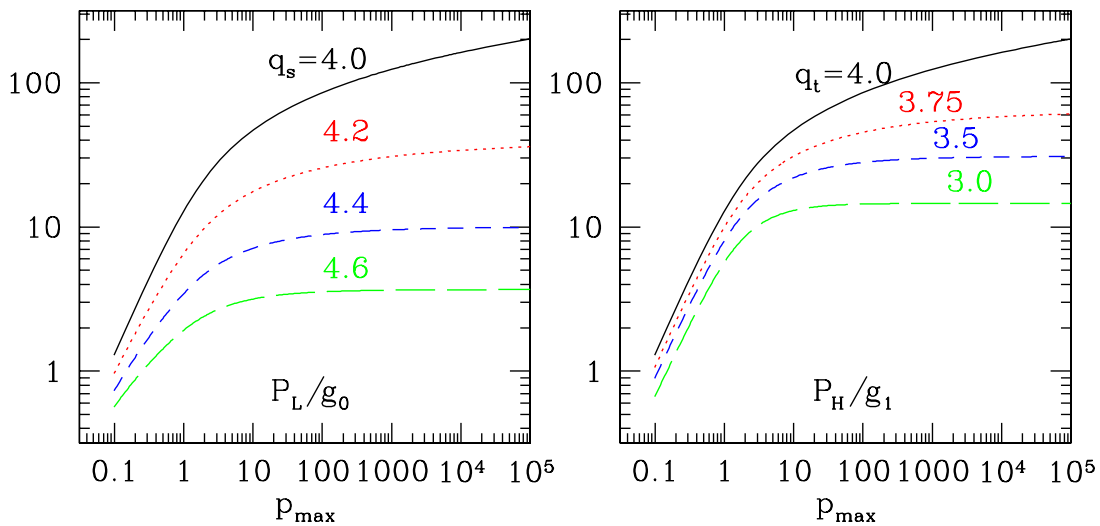

Figure 8. Left panel: $P_{L}$, defined in Equation (15), for $q_{s}=4.0-4.6$. Right panel: $P_{H}$, defined in Equation (15), for $q_{t}=3.0-4.0$. Here the injection momentum is $p_{\text {inj }}=10^{-2}$.

(A color version of this figure is available in the online journal.)
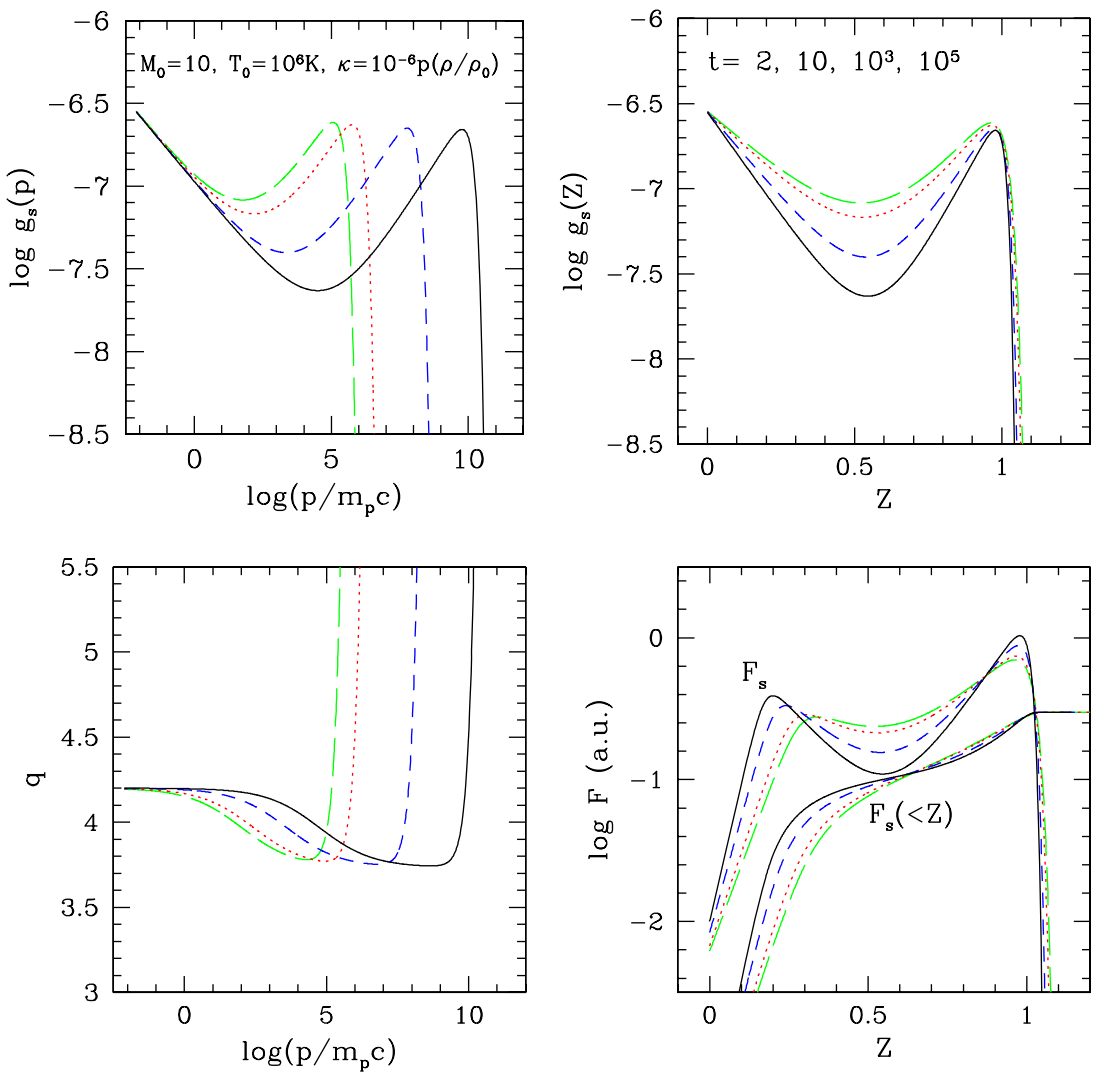

Figure 9. Upper panels: CR distribution at the subshock calculated using the analytic fitting formula in Equation (14) with $q_{s}=4.20, q_{t}=3.76, p_{\text {inj }}=10^{-2}$, and $p_{\max }=10^{5} t$. Lower panels: power-law slope of the fitted $g_{s}$, i.e., $q=-d \ln g_{s} / d \ln p+4$, and partial pressure, $F_{s}(Z)$, and its cumulative distribution, $F_{s}(<Z)$.

(A color version of this figure is available in the online journal.)

can see that $P_{c, 2}$ stays almost constant with the constant value of $g_{1}$, even though $p_{\max }$ increases 5 orders of magnitude. In fact, $P_{c, 2} /\left(\rho_{0} u_{s, i}^{2}\right)$ increases from 0.30 to 0.32 as $p_{\max }$ increases from $10^{5}$ to $10^{10}$. For such a long span of time, however, $g_{s}(Z)$ or $F_{s}(Z)$ does not keep the same shape. At $t=10^{5}$, the maximum momentum corresponds to $p_{\max } \approx 10^{19}(\mathrm{eV} / c)$ for protons.

One might ask how we can justify the validity of the proposed form of $g_{s}$ at $t \gg 1$, while our DSA simulations have been carried up to $t \sim 10-20$. In the T6P1d model, $p_{\max } \sim 10^{6}$ at $t=10$. So, most CRs are already ultrarelativistic, and the CR spectrum evolves as expected (i.e., according to Equation (14)). As long as $P_{c, 2}$ stays constant, the self-similarity of the precursor/subshock structure would be preserved even for $t \gg 1$. The stretching of the $u(x)$ profile in the precursor should influence the slope of the CR spectrum in a selfconsistent way as shown in Figure 6. There is no physical reason why such feedback between the precursor structure and the CR spectrum cannot be extended to $t \gg 1$, as long as the assumed CR diffusion model remains valid and the most energetic particles remain contained within the system. In realistic shocks, however, the assumption for Bohm diffusion could break down due to inefficient generation of waves in the precursor. Moreover, highest energy particles escape from the system, when their diffusion length becomes larger that the physical extent of the shock. The effects of escaping particles will be explored further in the next section. 

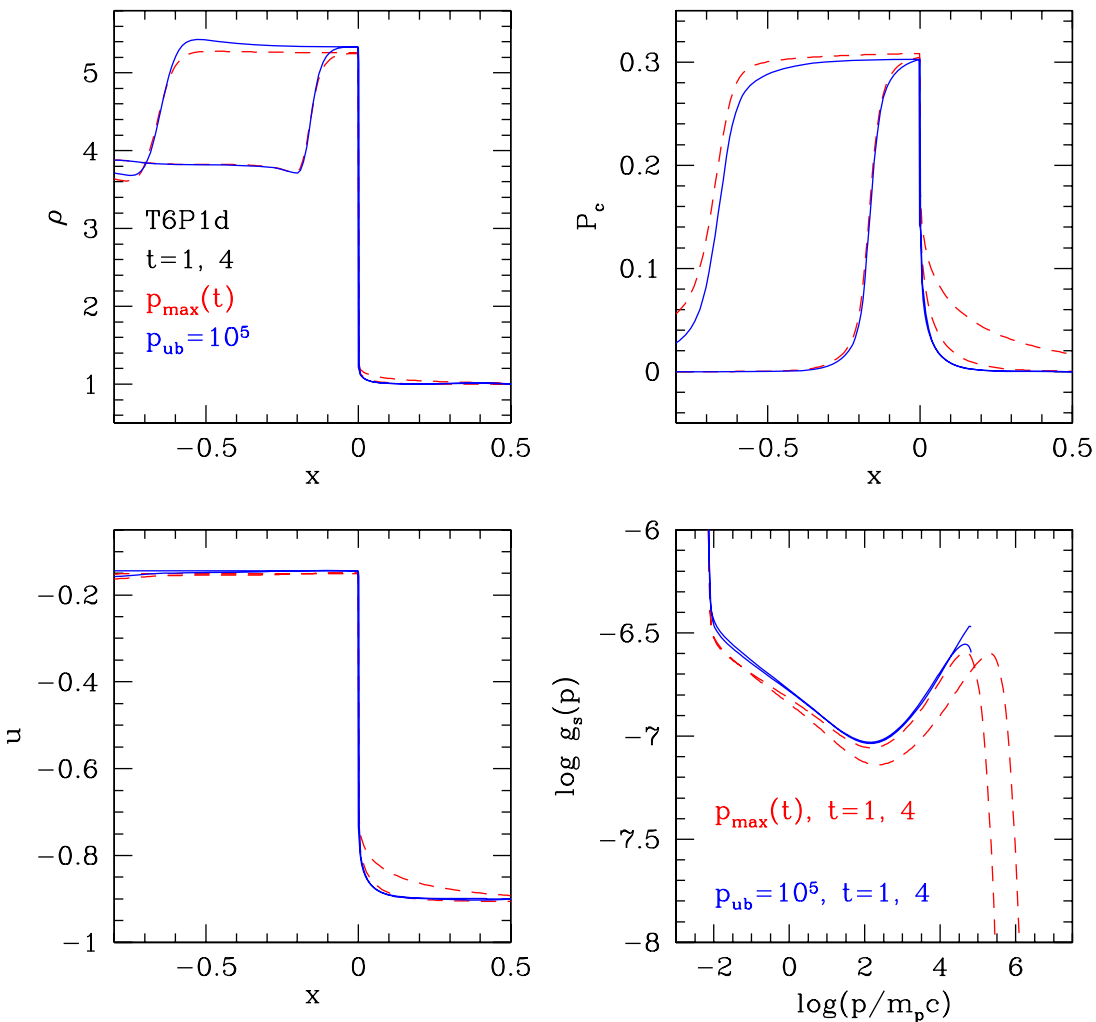

Figure 10. Comparison of the run with particle escape at $p_{\mathrm{ub}}=10^{5}$ (solid lines) and the run without particle escape (dashed line) for T6P1d model. The shock structure and the CR distribution are shown at $t=1$ and 4 .

(A color version of this figure is available in the online journal.)

We have focused here on moderately strong shock evolution with $M_{0} \gtrsim 10$, since it is much more complicated to study nonlinear DSA at weaker shocks with $M_{0}<10$. Nonrelativistic CRs play a more significant role within those shocks. For instance, since $P_{c}$ is not dominated by relativistic CRs, we need to follow more accurately the diffusion of nonrelativistic particles on scales close to the physical subshock thickness. Consequently, the diffusion model and the numerical grid resolution become important. The solutions also depend sensitively on the injection momentum, especially for shocks with Mach numbers, $M_{0} \lesssim 2.5$, where modifications are small, so the nearly test-particle CR spectrum is largely controlled by the injection momentum. Physics of thermal leakage injection, however, is not fully understood yet and we have only a working numerical model. Thus, we defer discussion of semianalytic discussion of evolving weak CR shocks to a separate paper.

\subsection{Steady State Shocks with a Fixed $p_{u b}$}

In realistic shocks, $p_{\max }(t)$ may reach an upper momentum boundary, $p_{\mathrm{ub}}$, beyond which CRs escape upstream from the shock due to the diffusion length, $l_{\max }$, approaching the physical size of the shocked system, or to lack of scattering waves at resonant scales of most energetic particles. From that time the precursor will cease to increase in scale and the self-similar evolution makes a transition into a stationary shock structure, or the one controlled by the overall dynamics of the situation. Because the shock energy is lost through particles escaping the system beyond $p_{\mathrm{ub}}$, the self-similar broadening of the precursor is replaced by a constant precursor structure in steady state.

We have calculated additional runs for the T6P1d model in which an upper momentum boundary condition, i.e., $g(p)=0.0$ for $p \geqslant p_{\mathrm{ub}}$ is enforced. In these simulations once $p_{\max }(t)$ has reached the given value of $p_{\mathrm{ub}}$, the highest energy particles escape from the shock, the CR spectrum becomes steady and the precursor stops growing. Figure 10 shows the results of T61Pd model with $p_{\mathrm{ub}}=10^{5}$ and without the upper momentum boundary. The distribution function $g_{s}(p)$ at the shock as well as the precursor and subshock structures all become steady after $t>1$ in the run with $p_{\mathrm{ub}}=10^{5}$. In the other run without particle escape, the precursor continues to broaden and $p_{\max }(t)$ increases with time. However, the postshock states (e.g., $\rho_{2}$ and $\left.P_{c, 2}\right)$ in the two runs are quite similar and $g_{s}(p)$ in the steady state limit is almost the same as that of the run without particle escape at $t \approx 1$, except the exponential tail above $p_{\max }$. In Figure 8 we showed that $P_{c, 2}$ stays constant as $p_{\max }(t)$ increases with time, if $g_{s}(p)$ follows the form given in Equation (14). This explains why $P_{c, 2}$ are very similar at different times in the two runs. Minor differences are slightly lower $P_{c, 2}$ and higher $\rho_{2}$ in the run with particle escape at $p_{\mathrm{ub}}$. We note that the compression ratio greater than 4 results mainly from the combined effect of the precursor compression and the subshock jump, i.e., $\sigma_{t}=\sigma_{p} \cdot \sigma_{s}$, regardless of particle escape. Energy loss due to escaping particles enhances the compression behind the shock only slightly in this shock, since the loss rate is not significant.

In Figures 11(a) and 11(b) snapshots are shown at $t=1$ for the runs with $p_{\mathrm{ub}}=10^{4}$ and $10^{5}$, and at $t=10$ for the run with $p_{\mathrm{ub}}=10^{6}$. For comparison, we also show the time-dependent solutions at $t=1$ and 10 for the run without particle escape, since in the evolving shock $p_{\max } \approx 10^{5}$ and $10^{6}$ at $t=1$ and 10 , respectively, for the T6P1d model. (At $t=0.1, p_{\max }$ would reach roughly to $10^{4}$, but by that time dynamical equilibrium has not been achieved and the self-similar evolution has not begun yet in the simulations.) The precursor structure shown in the 

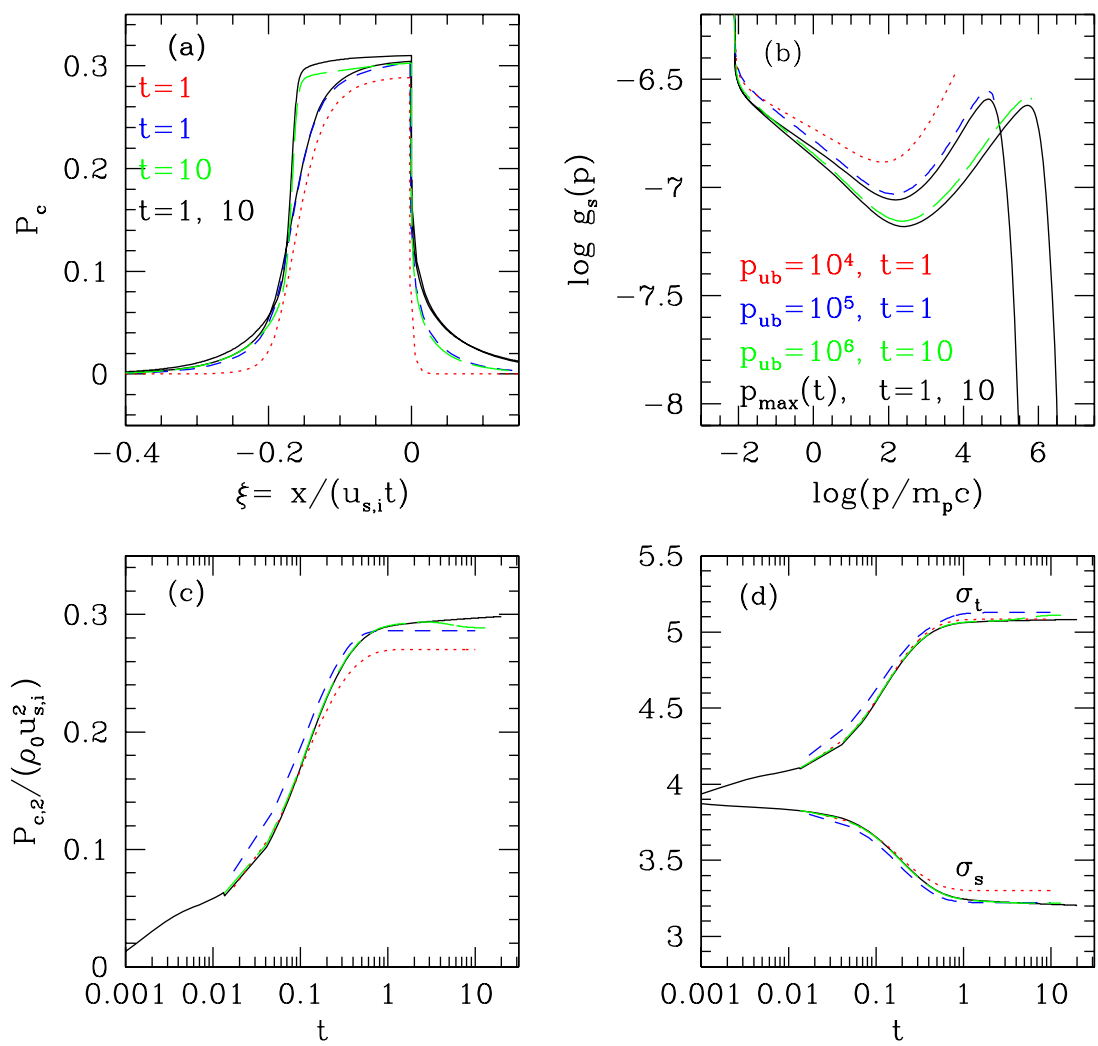

Figure 11. Comparison of the runs with and without particle escape at $p_{\mathrm{ub}}$ for T6P1d model. (a) CR pressure profiles in the three runs with $p_{\mathrm{ub}}=10^{4}$ at $t=1$ (dotted line), with $p_{\mathrm{ub}}=10^{5}$ at $t=1$ (dashed), and with $p_{\mathrm{ub}}=10^{6}$ at $t=10$ (long dashed line). The solid lines are for the run without particle escape at $t=1$ and 10 . (b) CR spectrum at the subshock. $(c)$ and $(d)$ Time evolution of the postshock CR pressure and the compression ratios. The same line types are used in all the panels. (A color version of this figure is available in the online journal.)

profile of $P_{c}$ reflects the diffusion length of highest momenta, $l_{d}\left(p_{\mathrm{ub}}\right) \propto p_{\mathrm{ub}}$ or $l_{d}\left(p_{\max }\right) \propto p_{\max }(t)$. Here the CR pressure is plotted against $\xi=x /\left(u_{s, i} t\right)$, since the results at two different times are shown together. So for example, the precursor width in $\xi$ is the same for the run with $p_{\mathrm{ub}}=10^{5}$ at $t=1$ (dashed line) and the run with $p_{\mathrm{ub}}=10^{6}$ at $t=10$ (long dashed line). Compared with these two runs, the run without particle escape at $t=1$ and 10 (solid lines) have a wider precursor due to the particles in the exponential tail above $p_{\max }(t)$. In Figures 11(c) and 11(d) we demonstrate that the evolution of the shock structure is quite similar and the shock approaches similar asymptotic states for all the runs, almost independent of $p_{\mathrm{ub}}$ or $p_{\max }(t)$, which is consistent with Figure 8. The asymptotic value of $P_{c, 2}$ is slightly lower and the precursor width is smaller in the runs with smaller $p_{\mathrm{ub}}$, as expected. Otherwise, the steady solutions with different $p_{\mathrm{ub}}$ are approximately the same as the time-dependent solutions at the time $t$ when $p_{\max }(t)$ equals to $p_{\mathrm{ub}}$. Thus, the proposed form of $g_{s}(p)$ can be applied to steady state shocks with an upper momentum boundary $p_{\mathrm{ub}}=p_{\max }$ as well, ignoring the exponential tail above $p_{\max }$. Even in the case where the shock structure is significantly affected by the energy loss due to escaping particles, Equation (14) can provide the steady state solution for $g_{s}(p)$, if the shock structures $\left(\sigma_{s}, \sigma_{t}\right.$ and postshock states) are known.

\section{SUMMARY}

We have studied the time-dependent evolution of the CR spectrum at CR-modified shocks in plane-parallel geometry, in which particles are accelerated to ever higher energies; that is, the maximum momentum $p_{\max }$ is not prefixed. We adopted Bohm diffusion as well as the diffusion with the power-law mo- mentum dependence of $\kappa(p) \propto p^{\alpha}$ with $0.5 \leqslant \alpha \leqslant 1$. Thermal leakage injection of suprathermal particles into the CR population at the subshock and finite Alfvén wave transport are included. Simulation parameters target nonrelativistic shocks with $M_{0} \gtrsim 10$ in warm photoionized and hot shock-heated astrophysical environments with magnetic field strengths somewhat below equipartition with the thermal plasma.

Unlike gasdynamic shocks, the time-asymptotic dynamical state of the evolving CR-modified shocks under consideration here cannot be found analytically either from the conservation equations or from the boundary conditions. So we rely on the kinetic simulations of diffusive shock acceleration to find the time-asymptotic state in the self-similar evolution stage. The general characteristics of the evolution of shock structure and particle spectrum can be summarized as follows.

1. The width of the precursor, $H$, scales with the diffusion length of the most energetic particles and for diffusion that scales as $\kappa=\kappa^{*}\left(\rho_{0} / \rho\right)^{v} p^{\alpha}$, increases linearly with time, i.e., $H \approx l_{\max } \approx 0.1 u_{s} t$, independent of the magnitude $\left(\kappa^{*}\right)$ and the value of $\alpha$.

2. If the acceleration timescale to reach relativistic energies from injection is much shorter than the dynamical timescale of the shock system (i.e., $\kappa^{*} \ll 0.1 u_{s} R$, where $R$ is the characteristic size of the shock), the $\mathrm{CR}$ pressure at the subshock approaches a constant value as the $P_{c}$ at the shock becomes a significant fraction of the momentum flux through the shock, $\sim \rho_{0} u_{0}^{2}$. For typical nonrelativistic shocks associated with cosmic structure, this transition roughly corresponds to a time when $p_{\max }$ becomes ultrarelativistic. Once this dynamical equilibrium develops, the shock precursor compression and the subshock jump are steady, leading to a self- 

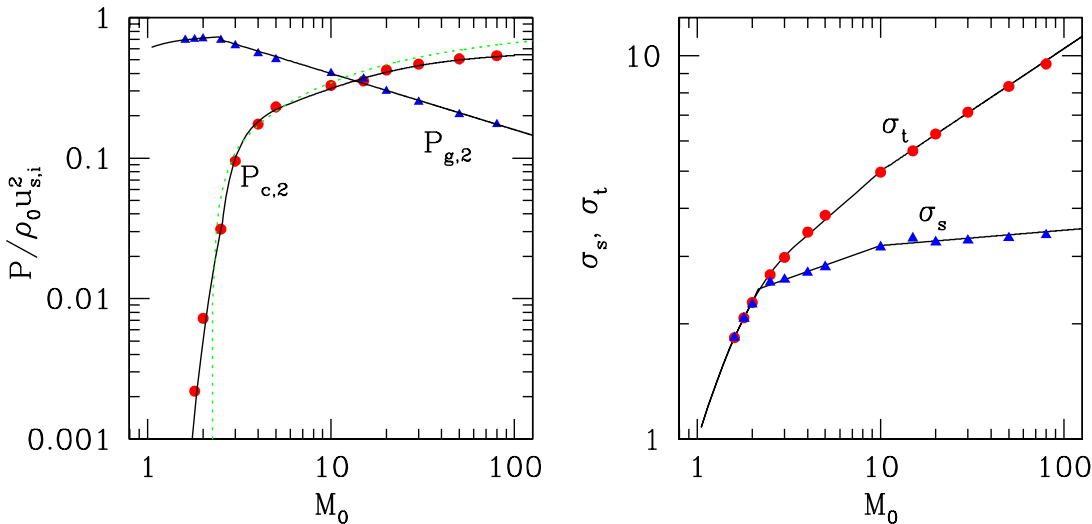

Figure 12. Time-asymptotic values of postshock gas and CR pressures in units of initial shock ram pressure (left panel), subshock compression ratio (triangles, right panel), and total compression ratio (circles, right panel) as a function of initial shock Mach number $M_{0}$ for T6P1d models. The solid lines show our fitting formulas given in Equations (A8)-(A11). The dotted line shows the estimate given in Equation (A7), adopting the numerical values of $\sigma_{p}=\sigma_{t} / \sigma_{s}$ in Equations (A10) and (A11).

(A color version of this figure is available in the online journal.)

similar stretching of the precursor with time. Consequently, the subshock compression ratio, $\sigma_{s}$, the total compression ratio, $\sigma_{t}$, as well as the postshock gas and CR pressures, $P_{g, 2}$ and $P_{c, 2}$, remain constant during the self-similar stage of the shock.

3. The lowest energy particles diffuse on a scale $l_{\min }=$ $\kappa\left(p_{\text {inj }}\right) / u_{s}$ and, so, experience only the compression across the subshock. Thus, near the injection momentum, $p_{\text {inj }}$, the CR distribution function is given by $f(p) \approx$ $f_{s, \text { th }}\left(p_{\text {inj }}\right)\left(p / p_{\text {inj }}\right)^{-q_{s}}$ where $f_{s, \text { th }}$ is the thermal Maxwellian distribution of the postshock gas and $q_{s}=3 \sigma_{s} /\left(\sigma_{s}-1\right)$. The amplitude $f_{\text {th }}\left(p_{\text {inj }}\right)$ is determined by the thermal leakage injection physics, since that establishes $p_{\text {inj }}$.

4. The most energetic particles diffuse on a scale $l_{\max }=$ $\kappa\left(p_{\max }\right) / u_{s}$ and, so, experience the total compression across the entire shock structure. Consequently, near $p_{\max }, f(p)$ flattens to $\left(p / p_{\max }\right)^{-q_{t}}$, where $q_{t}=3 \sigma_{t} /\left(\sigma_{t}-1\right)$. For $p>p_{\max }, f(p)$ is suppressed by an exponential cutoff.

Considering these facts, we proposed that the CR spectrum at the subshock for arbitrary time $t$ after self-similar evolution begins can be described approximately by the following simple analytic formula:

$$
\begin{aligned}
f_{s}(p, t)= & {\left[f_{0} \cdot\left(\frac{p}{p_{\text {inj }}}\right)^{-q_{s}}+f_{1} \cdot\left(\frac{p}{p_{\max }(t)}\right)^{-q_{t}}\right] } \\
& \times \exp \left[-\left(\frac{p}{1.5 p_{\max }(t)}\right)^{2 \alpha}\right],
\end{aligned}
$$

where $f_{0}=f_{s, \mathrm{th}}\left(p_{\text {inj }}\right)$ and $p_{\max } \propto\left(u_{s}^{2} t / \kappa^{*}\right)^{1 / \alpha}$ is given in Equation (10). The parameters, $p_{\mathrm{inj}}, q_{s}$, and $q_{t}$ can be estimated from the shock structure in the self-similar stage using DSA simulations results as outlined in the Appendix. The amplitude, $f_{1}$, has to satisfy the relation $g_{s}\left(p_{\max }\right)=f_{s}\left(p_{\max }\right) p_{\max }^{4} \approx$ constant in order for the postshock $P_{c}$ to remain steady. So, the momentum distribution function $g(p)$ is shifted to higher $p_{\max }$ in time, while keeping the amplitude at $p_{\max }$ constant in the self-similar stage. Hence, $p_{\max }$ is the only time-dependent parameter in Equation (16).

In a realistic shock geometry, however, CRs may escape upstream from the shock due to largest diffusion length approaching the physical size of the shocked system, or due to lack of scattering waves at resonant scales of most energetic particles. Once $p_{\max }$ approaches some upper momentum boundary at $p_{\text {up }}$, the shock structure and the CR spectrum develop steady states that are approximately the same as the evolving forms with $p_{\text {max }}=p_{\text {up }}$, except that some differences in the shock structure due to energy loss from escaping particles. Otherwise, the shock structure parameters and the approximate analytic form for the CR spectrum in the self-similar stage are consistent with previously proposed analytic and semianalytic steady state solutions (e.g., Berezhko \& Ellison 1999; Amato \& Blasi 2005).

Finally, we note that the evolution of the CR spectrum is secular in terms of the variable, $Z=\ln \left(p / p_{\text {inj }}\right) / \ln \left(p_{\max } / p_{\text {inj }}\right)$, which alluded wrongfully the self-similar evolution of the partial pressure function $F_{s}(Z)$ in Paper I. In fact, there is no similarity relation between $p$ and $t$.

H.K. was supported by the Korea Research Foundation Grant funded by the Korean Government (MOEHRD; R04-2006-000100590). D.R. was supported by the Korea Research Foundation Grant funded by the Korean Government (MOEHRD; KRF2007-341-C00020). T.W.J. is supported at the University of Minnesota by NASA grant NNG05GF57G, NSF grant Ast0607674 and by the Minnesota Supercomputing Institute.

\section{APPENDIX}

\section{ANALYTIC APPROXIMATIONS FOR DYNAMICAL STATES}

As we noted in Section 1, there are several analytic and semianalytic treatments of strong, steady state CR-modified shocks. The full time-asymptotic state of evolving CR-modified shocks can be obtained only through numerical simulations of nonlinear DSA. However, such simulations show strong similarities between steady state and asymptotic, evolving shocks. Here we outline some of those basic dynamical relations as they can be estimated analytically and empirically from our simulations, as reported in this paper and previously in Paper I.

A key to this comparison is the fact that the timescale for evolution of the shock precursor is the acceleration timescale to reach $p_{\max }, t_{\mathrm{acc}} \sim 10\left(l_{\max } / u_{s}\right)$ (see Equation (9)), which is characteristically an order of magnitude greater than the timescale for a fluid element to pass through the precursor, $t_{\text {dyn }} \sim l_{\max } / u_{s}$. Then, in following a fluid element through the precursor, one can neglect terms $\partial / \partial t$ compared with terms $u \partial / \partial x$ in evaluating the Lagrangian time variation, $d / d t$. For 
example, Equation (3), which can be expressed as

$$
\frac{d}{d t}\left(\frac{P_{g}}{\rho^{5 / 3}}\right)=\frac{2}{3} \frac{W}{\rho^{5 / 3}},
$$

assuming $\gamma_{g}=5 / 3$, then gives for an evolving precursor

$$
P_{g, 1} \approx\left(P_{g, 0}+\frac{2}{5} \rho_{0} u_{0}^{2} I\right) \sigma_{p}^{5 / 3}
$$

where $\sigma_{p}=\rho_{1} / \rho_{0}$ is the precursor compression factor. The quantity

$$
I=\frac{5}{3 u_{0}^{3} \rho_{0}^{1 / 3}} \int \frac{|W|}{\rho^{2 / 3}} d x
$$

was introduced in Paper I, and measures entropy added by Alfvén wave dissipation while the fluid element crosses the precursor, normalized by $u_{0}^{2} \rho_{0} / \rho_{0}^{5 / 3}$. Since Equation (A2) applies to an evolving shock, the subscripts " 0 " and " 1 " refer to states of a given fluid element as it enters the precursor and as it reaches the subshock. The approximation comes from neglecting explicit time variations in $|W|$ and $\rho$ in evaluating $I$. Equation (A2) is exact for a steady state shock. In the absence of Alfvén wave dissipation, this equation simply states the properties of adiabatic compression through the precursor, which obviously does not depend on the precursor being steady state.

Along similar lines, momentum conservation of a fluid element passing through the (slowly) evolving precursor gives

$$
P_{c, 1}+P_{g, 1} \approx P_{g, 0}+\rho_{0} u_{0}^{2}\left(1-\frac{1}{\sigma_{p}}\right),
$$

which can be combined with Equation (A2) to produce a simple estimate for the CR pressure at the subshock,

$$
P_{c, 1}=P_{c, 2} \approx \rho_{0} u_{0}^{2}\left[1-\frac{1}{\sigma_{p}}-\frac{3}{5} \frac{\sigma_{p}^{5 / 3}-1}{M_{0}^{2}}-\frac{2}{5} I \sigma_{p}^{5 / 3}\right] .
$$

By substituting Equation (A5) into Equation (A3) along with Equation (4), one can obtain

$$
I \approx \frac{5}{3} \frac{v_{A, 0}}{u_{0}} \frac{P_{c, 1}}{\rho_{0} u_{0}^{2}}
$$

where, once again, the approximation reflects neglect of explicit time variation in the shock structure during passage of a fluid element through the shock. Substituting this back into Equation (A5) we obtain

$$
\frac{P_{c, 2}}{\rho_{0} u_{0}^{2}} \approx\left[1-\frac{1}{\sigma_{p}}-\frac{3}{5} \frac{\sigma_{p}^{5 / 3}-1}{M_{0}^{2}}\right]\left[1+\frac{2}{3} \frac{v_{A, 0}}{u_{0}} \sigma_{p}^{5 / 3}\right]^{-1} .
$$

Given $P_{c, 1}=P_{c, 2}$ from Equation (A7) and using Equation (A4) it is straightforward to determine, as well, $P_{g, 1}$.

Although we can estimate approximately the postshock pressures, $P_{g, 2}$ and $P_{c, 2}$, for a given value of precursor compression, we must rely on numerical simulations to obtain the value of $\sigma_{p}$ for different model parameters. In the remainder of this appendix, we present some practical expressions for the shock dynamical properties obtained in our DSA simulations using a wide range of Mach numbers for the thermal injection parameter $\epsilon_{B}=0.2$, the Alfvén wave transport parameter $\theta=0.1$, and the diffusion coefficient $\kappa=\kappa^{*} p\left(\rho / \rho_{0}\right)$. In Figure 11, the timeasymptotic values of postshock CR pressure, gas pressure, and compression ratios are plotted against the initial shock Mach number $\left(M_{0} \geqslant 1.5\right)$.

For $M_{0} \leqslant 2.5$, the CR modification is negligible, so the postshock gas pressure and the shock compression ratios $\sigma_{t}=$ $\sigma_{s}$ are given by the usual Rankine-Hugoniot relation for pure gasdynamic shocks.

For $M_{0}>2.5$, the numerical results for the postshock gas pressure can be fitted by

$$
\frac{P_{g, 2}}{\rho_{0} u_{s, i}^{2}} \approx 0.4\left(\frac{M_{0}}{10}\right)^{-0.4} .
$$

The time-asymptotic density compression ratios can be approximated as follows:

$$
\begin{aligned}
& \sigma_{s} \approx 3.2\left(\frac{M_{0}}{10}\right)^{0.17} \text { for } 2.5 \leqslant M_{0} \leqslant 10, \\
& \sigma_{s} \approx 3.2\left(\frac{M_{0}}{10}\right)^{0.04} \text { for } M_{0}>10, \\
& \sigma_{t} \approx 5.0\left(\frac{M_{0}}{10}\right)^{0.42} \text { for } 2.5 \leqslant M_{0} \leqslant 10, \\
& \sigma_{t} \approx 5.0\left(\frac{M_{0}}{10}\right)^{0.32} \text { for } M_{0}>10 .
\end{aligned}
$$

We note that the subshock compression depends only weakly on $M_{0}$, while the total compression increases approximately as $M_{0}^{1 / 3}$. Even for strong shocks with $M_{0}$ up to 100 , the total compression ratio is less than 10 , because the propagation and dissipation of Alfvén waves upstream reduces the $\mathrm{CR}$ acceleration and the precursor compression.

The postshock CR pressure can be fitted empirically as follows:

$$
\begin{aligned}
& \frac{P_{c, 2}}{\rho_{0} u_{s, i}^{2}} \approx 2.34 \times 10^{-2}\left(M_{0}-1\right)^{3} \text { for } 1.5<M_{0}<2.5, \\
& \frac{P_{c, 2}}{\rho_{0} u_{s, i}^{2}} \approx \frac{0.58\left(M_{0}-1\right)^{4}}{M_{0}^{4}}-\frac{2.14\left(M_{0}-1\right)^{3}}{M_{0}^{4}}+\frac{13.7\left(M_{0}-1\right)^{2}}{M_{0}^{4}}(\mathrm{~A} 11) \\
& -\frac{27.0\left(M_{0}-1\right)}{M_{0}^{4}}+\frac{15.0}{M_{0}^{4}} \text { for } 2.5 \leqslant M_{0} \leqslant 100, \\
& \frac{P_{c, 2}}{\rho_{0} u_{s, i}^{2}} \approx 0.55 \text { for } M_{0}>100 .
\end{aligned}
$$

These fits are plotted as solid lines in Figure 12. Since $\sigma_{p}=$ $\sigma_{t} / \sigma_{s}$, Equations (A9) and (A10) can be used along with Equation (A7) to estimate $P_{c, 2}$ (dotted line in Figure 12).

In Kang et al. (2002), we showed that the effective injection momentum is $p_{\text {inj }} / p_{\text {th }} \approx 2.5$ for $M_{0} \gtrsim 10$ for the injection parameter $\epsilon_{B}=0.2$, where $p_{\text {th }}=2 \sqrt{k T_{2} / m_{p} c^{2}}$, and $T_{2}=$ $\left(P_{g, 2} / \rho_{2}\right)\left(m_{p} / k\right)$ is the postshock gas temperature. Then the thermal distribution at the injection momentum, $g_{s, \text { th }}\left(p_{\text {inj }}\right)$, can be calculated from the Maxwell distribution, since the postshock gas states, $T_{2}$ and $\rho_{2}$, are known. 


\section{REFERENCES}

Achterberg, A. 1982, A\&A, 98, 195

Achterberg, A., \& Blandford, R. D. 1986, MNRAS, 218, 551

Amato, E., \& Blasi, P. 2005, MNRAS, 364, L76

Amato, E., \& Blasi, P. 2006, MNRAS, 371, 1251

Bell, A. R. 1978, MNRAS, 182, 147

Bell, A. R. 2004, MNRAS, 353, 550

Berezhko, E. G., \& Ellison, D. C. 1999, ApJ, 526, 385

Berezhko, E. G., \& Völk, H. J. 1997, Astropart. Phys., 7, 183

Blandford, R. D., \& Eichler, D. 1987, Phys. Rep., 154, 1

Drury, L. O'C. 1983, Rep. Prog. Phys., 46, 973

Drury, L. O'C. 1984, Adv. Space Res., 4, 185

Drury, L. O'C., Axford, W. I., \& Summers, D. 1982, MNRAS, 198, 833
Duffy, P., Drury, L. O'C., \& Völk, H. 1994, A\&A, 291, 613

Jones, T. W. 1993, ApJ, 413, 619

Kang, H. 2003, J. Korean Astron. Soc., 36, 1

Kang, H., \& Jones, T. W. 2005, ApJ, 620, 44

Kang, H., \& Jones, T. W. 2006, Astropart. Phys., 25, 246

Kang, H., \& Jones, T. W. 2007, Astropart. Phys, 28, 232 (Paper I)

Kang, H., Jones, T. W., \& Gieseler, U. D. J. 2002, ApJ, 579, 337

Lucek, S. G., \& Bell, A. R. 2000, MNRAS, 314, 65

Malkov, M. A. 1997, ApJ, 485, 638

Malkov, M. A. 1999, ApJ, 511, L53

Malkov, M. A., \& Drury, L. O'C. 2001, Rep. Prog. Phys., 64, 429

Malkov, M. A., \& Völk, H. J. 1998, Adv. Space Res., 21, 551

Skilling, J. 1975a, MNRAS, 172, 557

Skilling, J. 1975b, MNRAS, 173, 255

Vladimirov, A., Ellison, D. C, \& Bykov, A. 2006, ApJ, 652, 1246 